\title{
Line and boundary tensions at the wetting transition: Two fluid phases on a substrate
}

Cite as: J. Chem. Phys. 102, 400 (1995); https://doi.org/10.1063/1.469416

Submitted: 11 August 1994 . Accepted: 19 September 1994 . Published Online: 04 June 1998

S. Perković, E. M. Blokhuis, and G. Han

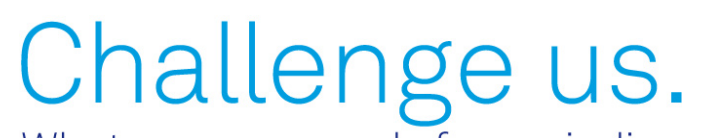

What are your needs for periodic

signal detection?
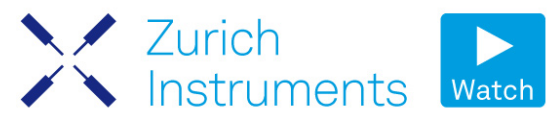

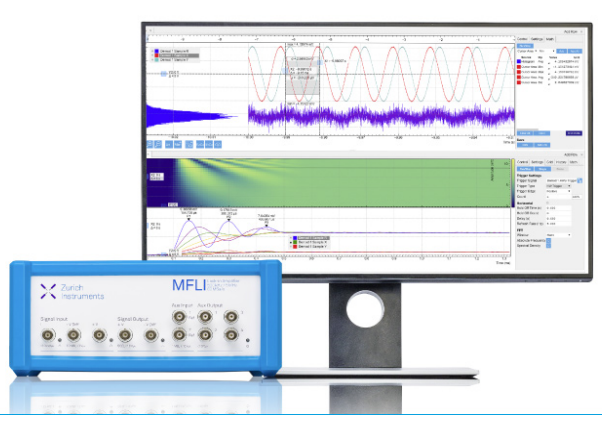

102,400 


\title{
Line and boundary tensions at the wetting transition: Two fluid phases on a substrate
}

\author{
S. Perković, E. M. Blokhuis, and G. Han \\ Department of Chemistry, Baker Laboratory, Cornell University, Ithaca, New York 14853
}

(Received 11 August 1994; accepted 19 September 1994)

\begin{abstract}
We develop and analyze a mean-field model free energy that describes two fluid phases on a substrate in order to calculate the (numerically) exact line and boundary tensions, on approach to the first-order wetting transition. A theory based on the van der Waals theory of gas-liquid interfaces is used. We implement a multigrid algorithm to determine the two-dimensional spatial variation of the density across the three-phase and boundary regions, and hence, the line and boundary tensions. As the wetting transition is approached, the tensions approach the same, finite, positive limit with diverging slopes. We compare our results with those of recent related work. (C) 1995 American Institute of Physics.
\end{abstract}

\section{INTRODUCTION}

Two bulk fluid phases $\alpha$ and $\beta$ on a substrate $\gamma$, at equilibrium, can meet at a common line of contact with nonzero contact angles ("partially wet" state) (Fig. 1). If the surface tension $\sigma^{\alpha \gamma}$ of the $\alpha \gamma$ interface is the largest of the three tensions $\sigma^{\alpha \gamma}, \sigma^{\alpha \beta}$, and $\sigma^{\beta \gamma}$, where $\sigma^{\alpha \beta}$ and $\sigma^{\beta \gamma}$ are the surface tensions of the $\alpha \beta$ and $\beta \gamma$ interfaces, respectively, the condition for mechanical equilibrium of the partially wet state is given by ${ }^{1}$

$$
\sigma^{\alpha \gamma}<\sigma^{\alpha \beta}+\sigma^{\beta \gamma}
$$

When the contact angle $\theta$ of the $\beta$ phase becomes 0 , the $\beta$ phase spreads on the $\gamma$ substrate (wet state) (Fig. 2). The equilibrium condition for the wet state is given by ${ }^{1}$

$$
\sigma^{\alpha \gamma}=\sigma^{\alpha \beta}+\sigma^{\beta \gamma}
$$

The transition between the former partially wet and the latter wet state is called a wetting transition. In Fig. 3, we show a generic phase diagram of a system of two fluid phases on a substrate that can undergo a first-order wetting transition. The variables $\mu_{1}$ and $\mu_{2}$ are any two thermodynamic fields, such as the temperature and the chemical potential. The solid curve represents states where the three bulk phases $\alpha, \beta$ and $\gamma$ coexist. The $W$ point denotes the wetting transition. Below it, on the coexistence curve, the partially wet states are thermodynamically stable. Above the $W$ point, on the coexistence curve, the wet states are stable. The first-order character of the wetting transition extends in the two-phase region to the left of the coexistence curve, where only the $\alpha$ and $\gamma$ phases are stable as bulk. There, the dashed curve represents states of coexistence of two surface phases-one that consists of a thin, microscopic layer of a $\beta$-like phase at the $\alpha \gamma$ interface and one where there is no such layer. This coexistence of two $\alpha \gamma$ interfaces, of different structure, but of equal tension, is called a prewetting transition. It is a first-order surface phase transition. At the prewetting transition, the surface tensions of the two surface phases are identical ${ }^{1}$

$$
\sigma^{\alpha \gamma}=\sigma^{\alpha \gamma^{*}}
$$

where $\sigma^{\alpha \gamma^{*}}$ is the surface tension of the $\alpha \gamma^{*}$ interface consisting of a thin $\beta$-like layer, and $\sigma^{\alpha \gamma}$ is the surface tension of the $\alpha \gamma$ interface where no such layer is present. The line of prewetting transitions (dashed curve in Fig. 3), called the prewetting line, meets the three-phase coexistence line tangentially. ${ }^{2}$ Along the prewetting line, as the wetting transition is approached, the $\beta$-like layer increases in thickness until it becomes macroscopically thick exactly at the wetting transition.

In the partially wet state, the three two-phase interfaces meet at a common line of contact - the three-phase contact line. The inhomogeneity in density associated with that line gives rise to an excess free energy over that in the bulk phases and in the interfaces. That excess free energy per unit length of the three-phase contact line is the line tension $\tau$. Related to $\tau$ is the boundary tension $\tau_{b}$. When two surface phases coexist at the prewetting transition, they do so by creating a one-dimensional boundary between them. The inhomogeneity in density associated with such a line gives rise to an excess free energy, which, per unit length of that line, defines the boundary tension.

The values of the line and boundary tensions at the wetting transition have been studied with different models, by several authors. ${ }^{3-8}$ Varea and Robledo ${ }^{7}$ studied a spin-1/2 Ising model within the mean-field approximation in a system where one of the phases was a wall. In their work, they solved the Euler-Lagrange equation for the magnetization, corresponding to minimizing the free energy of the system, and then obtained the line and boundary tensions. They conjectured that $\tau$ and $\tau_{b}$ diverge at the wetting transition. In a later work, using more precise calculations, they argued that $\tau_{b}$ is finite at the wetting transition, and approaches it with a diverging slope. ${ }^{8}$

At the same time, calculations of $\tau$ and $\tau_{b}$ for continuous systems have been performed with two different phenomenological approaches. At a more microscopic level, a phenomenological theory based on the van der Waals theory of gasliquid interfaces is used to calculate $\tau$ and $\tau_{b}$ in a system of fluid phases at coexistence. In this theory, the line tension $\tau$ is given by ${ }^{1}$

$$
\tau=\min _{\rho_{1}, \ldots, \rho_{n}} \lim _{R \rightarrow \infty}\left[\int_{A} d a \Psi-\left(\sigma^{\alpha \gamma}+\sigma^{\alpha \beta}+\sigma^{\beta \gamma}\right) R\right],
$$




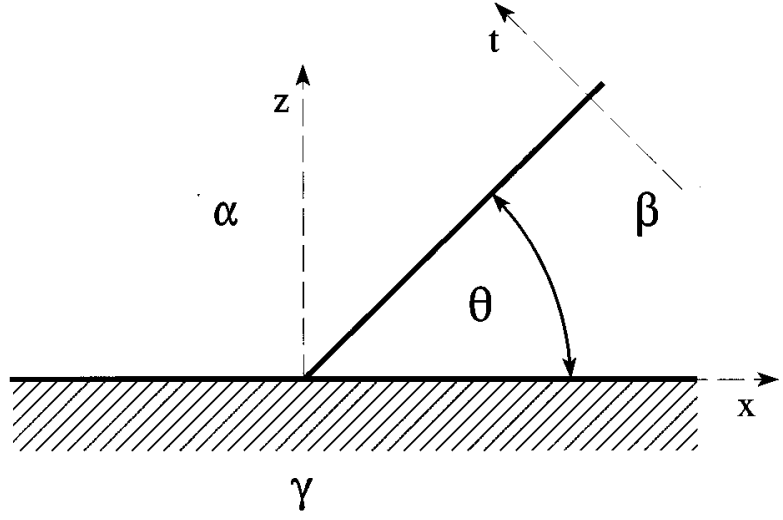

FIG. 1. A side view of two fluid phases $\alpha$ and $\beta$ on a substrate $\gamma$ in a partially wet state. $\theta$ is the contact angle that the $\beta$ phase forms with the $\gamma$ substrate. The three two-phase interfaces meet at the three-phase contact line.

where the integration is done over a large domain of area $A$ in a plane perpendicular to the three-phase contact line and whose sides are perpendicular to the three two-phase interfaces (see Fig. 7 in Sec. III). The distance $R$ is the length of the two-phase interfaces within the area $A$. In general these lengths are different for the $\alpha \gamma, \alpha \beta$, and $\beta \gamma$ interfaces, so a more general equation than Eq. (1.4) is available. However, for simplicity, we keep these three lengths equal. $\Psi$ is a local excess free-energy density that is a functional of the densities $\rho_{1}, \rho_{2}, \ldots, \rho_{n}$ of the system's $n$ components. It is assumed to be of the following form: ${ }^{1}$

$$
\Psi=F\left(\rho_{1}, \rho_{2}, \ldots, \rho_{n}\right)+\frac{1}{2} \sum_{i, j} m_{i, j} \nabla \rho_{i} \cdot \nabla \rho_{j},
$$

where $F$ is a local excess free-energy density in an environment of homogeneous density and the dot product of the gradient terms $\boldsymbol{\nabla} \rho_{i}$ and $\boldsymbol{\nabla} \rho_{j}$ describes the local excess freeenergy density due to the inhomogeneity in the densities in the interfacial and contact line regions. The gradient operator $\boldsymbol{\nabla}$ is two dimensional, in the plane perpendicular to the threephase contact line. There is no variation in the densities in the direction parallel to the contact line. The $m_{i, j}$ 's are con-

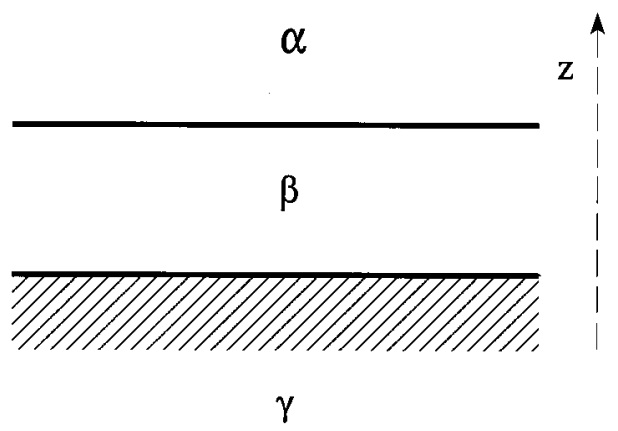

FIG. 2. A side view of two fluid phases $\alpha$ and $\beta$ on a substrate $\gamma$ in a wet state.

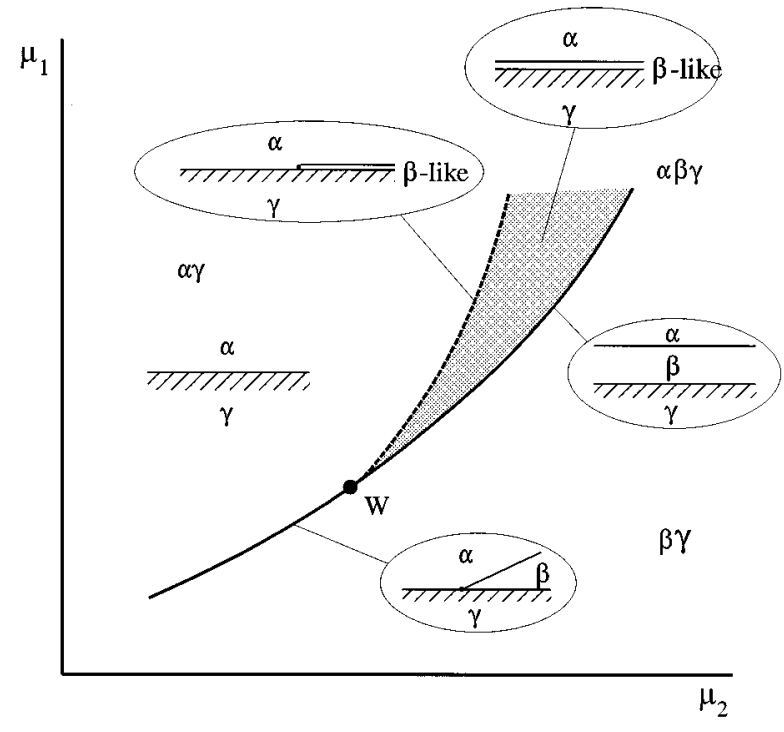

FIG. 3. A generic phase diagram of two fluid phases on a substrate. $\mu_{1}$ and $\mu_{2}$ are any two thermodynamic fields. The solid curve is the three-phase coexistence curve. The point $W$ represents the wetting transition. Below the point $W$, on the solid curve, the stable states are partially wet, and above it, they are wet. The dashed curve, called the prewetting line, represents the locus of prewetting transitions, where two different structures of the $\alpha \gamma$ interface coexist. The prewetting line meets the three-phase coexistence line tangentially at the wetting transition $W$.

stants and $\sigma^{\alpha \gamma}, \sigma^{\alpha \beta}$, and $\sigma^{\beta \gamma}$ are the surface tensions of the $\alpha \gamma, \alpha \beta$, and $\beta \gamma$ interfaces, respectively. They are defined, far from the three-phase contact line, as follows: ${ }^{1}$

$$
\sigma=\min _{\rho_{1}, \ldots, \rho_{n}} \int_{-\infty}^{\infty} d z \Psi
$$

where $\Psi$ is defined as in Eq. (1.5) and is a function of $z$ alone, where $z$ is the direction perpendicular to the individual interfaces. Therefore, the gradient operator $\boldsymbol{\nabla}$ is one dimensional in the $z$ direction.

Using the van der Waals theory, Szleifer and Widom ${ }^{5}$ calculated the line tension $\tau$ in a two-component system of three fluid phases at coexistence by describing the two densities with approximate, but qualitatively correct functional forms with variational parameters. The values of these parameters were determined by minimizing Eq. (1.4). They calculated $\tau$ as a function of the contact angle $\theta$ of the wetting phase, up to $\theta=13^{\circ}$. They argued that $\tau$ possibly diverges as $1 / \theta$, as the wetting transition is approached $(\theta \rightarrow 0)$.

By adding a positive thermodynamic field $\epsilon$ to describe the deviation of the system from three-phase coexistence, Perkovic, Szleifer, and Widom ${ }^{4}$ extended the model of Szleifer and Widom to calculate the boundary tension $\tau_{b}$ as the wetting transition is approached along the prewetting line. Within the van der Waals theory, the boundary tension $\tau_{b}$ is given $b^{9}$

$$
\tau_{b}=\min _{\rho_{1}, \ldots, \rho_{n}} \int_{-\infty}^{\infty} d x\left[\int_{-\infty}^{\infty} d z(\Psi)-\sigma\right]
$$




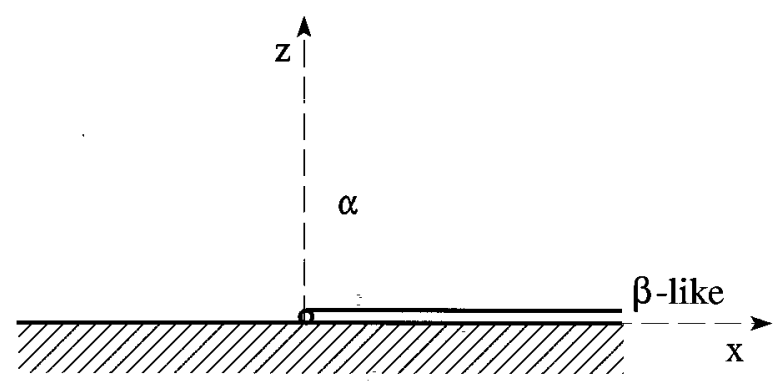

$\gamma$

FIG. 4. A side view of two surface phases coexisting at the prewetting transition. On the right, a microscopic layer of a $\beta$-like phase spreads at the $\alpha \gamma$ interface, while no such layer exists at the $\alpha \gamma$ interface on the left. The two interfaces meet edge on to create the boundary line.

where $\Psi$ is given by Eq. (1.5) and $\sigma$ by Eq. (1.6). It should be noted that $\Psi$ is now a function of the coordinates $x$ and $z$ perpendicular to the boundary line (Fig. 4). The same approximate method was used to calculate $\tau_{b}$. The values of the boundary tension $\tau_{b}$ showed an apparent finite limit at the wetting transition, in contrast to the apparent divergence of the line tension $\tau$ from Ref. 5 .

Another, more phenomenological approach for determining $\tau$ and $\tau_{b}$ at the wetting transition is the interface displacement model. ${ }^{6,10}$ This model defines the interface displacement $l(x)$ as the height above a solid substrate, where the density profile of a one-component, liquid-vapor system equals a certain fixed value. The interface displacement $l(x)$ is then a measure of the thickness of the liquid layer on the substrate. The boundary tension $\tau_{b}$ is given by the following expression: ${ }^{6}$

$$
\tau_{b}=\min _{l} \int_{-\infty}^{\infty} d x\left[\frac{\sigma_{0}}{2}\left(\frac{d l}{d x}\right)^{2}+V(l)+\mathrm{const}\right] .
$$

An analogous expression exists for the line tension $\tau$. The first term in the integrand of Eq. (1.8) describes the increase of the excess free energy per unit area due to an increase of interfacial area, for small interface displacement gradients; $\sigma_{0}$ is the surface tension of the two-fluid interface and $V(l)$ is an excess free energy per unit area. It has its minimum values at $V\left(l=l_{\text {thin }}\right)$ and $V\left(l=l_{\text {thick }}\right)$, where $l_{\text {thin }}$ and $l_{\text {thick }}$ are the thicknesses of the thin and thick layers far from the boundary region. The $x$ coordinate is parallel to the substrate. The constant is chosen such that the integrand in Eq. (1.8) vanishes as $x \rightarrow \pm \infty$. Indekeu ${ }^{6}$ determined that at the first-order wetting transition, for short-range forces, the line and boundary tensions are positive and finite, approaching the wetting transition with a slope diverging as a function of an appropriate thermodynamic field that measures the distance from the wetting transition.

The interface displacement model has been applied successfully in determining the tensions beyond the gradientsquare approximation ${ }^{11}$ and near multicritical wetting transitions, ${ }^{12}$ as well as for exploring the universal properties of the first-order wetting transition. ${ }^{13}$ However, it is a more phenomenological approach ${ }^{3,6}$ than the van der Waals theory. Only recently did Blokhuis ${ }^{3}$ make a connection between the more fundamental van der Waals approach and the interface displacement model, by studying the expression for the boundary tension $\tau_{b}$. The model free energy that he used is an extension of the van der Waals expression (1.7) to a system that contains a substrate as the third phase. ${ }^{14}$ Blokhuis derived explicit expressions for the functions $\sigma_{0}(l)$ (not a constant, as was assumed in Ref. 6) and $V(l)$ in terms of the density profile of the one component system. The boundary tension was determined using approximate forms for the density profile. A positive, finite upper bound to $\tau_{b}$ was obtained at the wetting transition, with the same asymptotic form for $\tau_{b}$ near the wetting transition as in the interface displacement model. Recently, Blokhuis ${ }^{15}$ determined, with the same model but applied to the partially wet case, the same functional form for the line tension $\tau$ near the wetting transition as in the interface displacement model, and then a finite, positive $\tau$ at the wetting transition.

In this paper, we describe the calculation of the (numerically) exact line tension of the three-phase contact line formed by two fluids on a substrate, by constraining the model free energy in Ref. 3 to a three-phase equilibrium. The presence of the substrate is treated as a boundary condition, which converts the system into a one-component one. Furthermore, we use the model free energy in Ref. 3 to calculate the exact boundary tension of the one-dimensional boundary formed when two surface phases coexist. These results represent the first exact calculations of the line and boundary tensions for a continuous system. Fitting the data with functional forms obtained by Indekeu ${ }^{6}$ and Blokhuis, ${ }^{3}$ we find that, at the wetting transition, the line and boundary tensions are positive and finite. Within the numerical accuracy, these two values are equal, as predicted by Widom. ${ }^{16}$ Furthermore, the line and boundary tensions are lower than the upper bounds for $\tau_{b}$ (Ref. 3) and for $\tau$, determined from the approximate calculations.

In the next section, we define the model free energy that we use to obtain the exact expressions for the interfacial density profiles and the surface tensions of the three twophase interfaces, and the density profiles and surface tensions of the two surface phases coexisting at the prewetting transition. In Sec. III, we calculate the line and boundary tensions using a multigrid algorithm. In Sec. IV, we calculate the functions $\sigma_{0}(l)$ and $V(l)$, defined in the interface displacement model, from our numerically obtained density profiles. We end, in Sec. V, with a discussion of the results.

\section{SURFACE TENSION}

In this section, we investigate the structures and tensions of the three interfaces far away from the three-phase contact region, when three bulk phases coexist in a partially wet state. We determine, as well, the profiles and tensions of the two surface phases that coexist at the prewetting transition. A model free energy that is an extension of the van der Waals expression (1.6) to a system that contains a substrate as the third phase is used. ${ }^{3,14}$ 
For a one-component, two fluid-phase system (the $\alpha$ and $\beta$ phases in Fig. 1, far from the solid substrate), the surface tension or excess free energy per unit area $\sigma^{\alpha \beta}$ of the $\alpha \beta$ interface is given by [cf. Eq. (1.6)]

$$
\sigma^{\alpha \beta}=\min _{\rho}^{\infty} \int_{-\infty}^{\infty} d t \Psi(\rho),
$$

where $\Psi(\rho)$ is a local excess free-energy density that is a functional of the local density $\rho=\rho(t)$, and is assumed to be of the following form [cf. Eq. (1.5)]:

$$
\Psi(\rho)=f(\rho)+\frac{1}{2}(\nabla \rho)^{2},
$$

with $f(\rho)$ given by

$$
f(\rho)=\frac{1}{2}\left(\rho^{2}-1\right)^{2} .
$$

The gradient operator $\boldsymbol{\nabla}$ is one dimensional in the $t$ direction which is perpendicular to the $\alpha \beta$ interface. There is no variation in the density in the directions parallel to the interface. The distance $t$, gradient operator $\nabla$, density $\rho$, and free energies $\sigma^{\alpha \beta}, \Psi$, and $f$ are scaled appropriately so that they are all dimensionless. The density $\rho$ is a relative density and so can be negative. At $\alpha \beta$ coexistence, the density of the $\alpha$ and $\beta$ phases are $\rho^{\alpha}=-1$ and $\rho^{\beta}=1$.

When a solid substrate is present as the third phase (the $\gamma$ phase in Fig. 1), the surface tension or excess free energy per unit area $\sigma^{\mathrm{fs}}$ of the fluid-solid interface, far from the three-phase contact line, is given by ${ }^{3,14}$

$$
\sigma^{f s}=\min _{\rho} \int_{0}^{\infty} d z[\Psi(\rho)]+\Phi\left(\rho_{1}\right),
$$

where $\Psi(\rho)$ is given in Eq. (2.2) and $\Phi\left(\rho_{1}\right)$ is a phenomenological term that accounts for the fluid-solid interactions and is assumed to be of the following form: ${ }^{14}$

$$
\Phi\left(\rho_{1}\right)=-h_{1} \rho_{1}-\frac{1}{2} g \rho_{1}^{2} .
$$

The density $\rho_{1} \equiv \rho(z=0)$ is the equilibrium density at the solid substrate, far from the three-phase contact region; $z$ is the coordinate perpendicular to the solid substrate; $\rho(z)$ is the local fluid density; and the phenomenological parameters $h_{1}$ and $g$ are the surface field and the surface enhancement parameters, respectively. The temperature dependence of $h_{1}$ and $g$ has been studied both theoretically ${ }^{14}$ and experimentally. ${ }^{17}$ We restrict ourselves to the case of $h_{1} \geqslant 0$ only. ${ }^{3}$ As in Eq. (2.1), the distances, densities, and energy densities in Eq. (2.4) are scaled so that they are all dimensionless.

For a system of two surface phases at coexistence (Fig. 4 ), the surface tension or the excess free energy per unit area $\sigma$ of the interfaces, far from the boundary region, is obtained as an extension of Eq. (2.4)

$$
\sigma=\min _{\rho} \int_{0}^{\infty} d z\left[\Psi_{b}(\rho)\right]+\Phi\left(\rho_{1}\right),
$$

where $\Phi\left(\rho_{1}\right)$ is given in Eq. (2.5) and $\Psi_{b}(\rho)$ is given by

$$
\Psi_{b}(\rho)=f(\rho)-f\left(\rho^{\alpha}\right)+\frac{1}{2}(\nabla \rho)^{2},
$$

with

$$
f(\rho)=\frac{1}{2}\left(\rho^{2}-1\right)^{2}+h \rho .
$$

The $\boldsymbol{\nabla}$ gradient operator in Eq. (2.7) is one dimensional in the $z$ direction, perpendicular to the substrate. The densities $\rho$ and $\rho_{1}$ are defined as in Eq. (2.4). The bulk field $h(h \geqslant 0)$ measures the distance from three bulk-phase coexistence. When $h=0$, the three phases $\alpha, \beta$ and $\gamma$ coexist with $\rho^{\alpha}=-1$ and $\rho^{\beta}=1$, while for $h>0$, only the $\alpha$ and $\gamma$ phases coexist. Then, the density of the $\alpha$ phase $\rho^{\alpha}$ is the density for which $f(\rho)$ is minimal. For small values of $h, \rho^{\alpha}$ is given by Eq. (2.3) in Ref. 3.

Far from the three-phase contact line (Fig. 1), the surface tension $\sigma^{\alpha \beta}$ of the $\alpha \beta$ interface is obtained by minimizing Eq. (2.1) with respect to the density $\rho$; i.e., by solving the Euler-Lagrange equation

$$
\frac{\partial^{2} \rho}{\partial t^{2}}=f^{\prime}(\rho)
$$

with the appropriate boundary conditions in the $\alpha$ and $\beta$ phases $\rho^{\alpha}=-1$ and $\rho^{\beta}=1$. The prime denotes the first derivative with respect to the argument.

The surface tensions $\sigma^{\alpha \gamma}$ and $\sigma^{\beta \gamma}$ of the $\alpha \gamma$ and $\beta \gamma$ interfaces are obtained by minimizing Eq. (2.4) with respect to the density $\rho$; i.e., by solving the Euler-Lagrange equation

$$
\frac{\partial^{2} \rho}{\partial z^{2}}=f^{\prime}(\rho)
$$

with the appropriate boundary conditions at the solid substrate

$$
\left.\frac{\partial \rho}{\partial z}\right|_{z=0}=\Phi^{\prime}\left(\rho_{1}\right)
$$

and in the $\alpha$ and $\beta$ phases $\rho^{\alpha}=-1$ and $\rho^{\beta}=1$.

The solutions to Eqs. (2.9) and (2.10) with the appropriate boundary conditions are the equilibrium density profiles, which can be determined analytically

$$
\begin{aligned}
& \rho^{\alpha \gamma}(z)=-\tanh \left(z-z_{0}\right), \\
& \rho^{\beta \gamma}(z)=\frac{1}{\tanh \left(z+z_{1}\right)}, \\
& \rho^{\alpha \beta}(t)=-\tanh (t),
\end{aligned}
$$

where the coordinate $t$ is defined as

$$
t \equiv-x \sin \theta+z \cos \theta .
$$

The coordinate $x$ is parallel to the solid substrate and $\theta$ is the contact angle that the $\beta$ phase forms with the substrate (Fig. 1).

The constants $z_{0}$ and $z_{1}$ are defined as functions of the model's two parameters $g$ and $h_{1}$,

$$
\begin{aligned}
& \tanh \left(z_{0}\right)=-\frac{1}{2} g-\frac{1}{2}\left(g^{2}-4 h_{1}+4\right)^{1 / 2}, \\
& \frac{1}{\tanh \left(z_{1}\right)}=\frac{1}{2} g+\frac{1}{2}\left(g^{2}+4 h_{1}+4\right)^{1 / 2} .
\end{aligned}
$$

Substituting the interfacial density profiles (2.14) in Eqs. (2.1) and (2.12) and (2.13) in Eq. (2.4), one obtains the surface tensions of the interfaces (far from the three-phase contact region) 


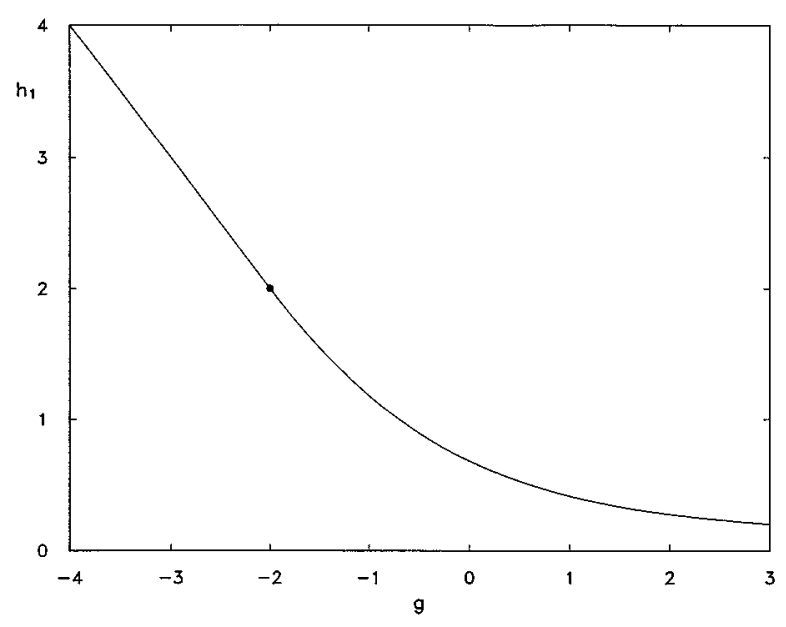

FIG. 5. The phase diagram of the model system studied, where $h_{1}$ and $g$ are the model's phenomenological parameters. The solid curve is the locus of wetting transitions. For $g>-2$, it is the locus of first order wetting transitions, while for $g<-2$, it is the locus of continuous wetting transitions. The point $g=-2, h_{1}=2$ is a (surface) tricritical point. In the region below the solid curve, partially wet states are stable. Above the solid curve, the wet states are stable.

$$
\begin{aligned}
\sigma^{\alpha \gamma} & =\frac{2}{3}-\frac{g}{12}\left(g^{2}-6 h_{1}+6\right)-\frac{1}{12}\left(g^{2}-4 h_{1}+4\right)^{3 / 2}, \\
\sigma^{\beta \gamma} & =\frac{2}{3}-\frac{g}{12}\left(g^{2}+6 h_{1}+6\right)-\frac{1}{12}\left(g^{2}+4 h_{1}+4\right)^{3 / 2}, \\
\sigma^{\alpha \beta} & =\frac{4}{3} .
\end{aligned}
$$

From Eqs. (2.18)-(2.20), using Young's equation, $\sigma^{\alpha y}=\sigma^{\beta \gamma}+\sigma^{\alpha \beta} \cos \theta$, to relate the contact angle $\theta$ to the surface tensions, the contact angle $\theta$ is

$\cos \theta=\frac{3}{4} g h_{1}+\frac{1}{16}\left[\left(g^{2}+4 h_{1}+4\right)^{3 / 2}-\left(g^{2}-4 h_{1}+4\right)^{3 / 2}\right]$.

By comparing the values of the two terms $\sigma^{\alpha \gamma}$ and $\sigma^{\alpha \beta}+\sigma^{\beta \gamma}$ at different values of $g$ and $h_{1}$, we are able to determine which of the two three-phase thermodynamic states is stable - the partially wet state or the wet state. The values of $g$ and $h_{1}$ for which $\sigma^{\alpha \gamma}=\sigma^{\alpha \beta}+\sigma^{\beta \gamma}$ determine the wetting transition. In Fig. 5, we show the phase diagram of this three bulk phase system. In the region below the solid curve, partially wet states are thermodynamically stable, while above the solid curve, wet states are the thermodynamically stable ones. The solid curve itself is the locus of wetting transitions. For $g>-2$, it is the locus of first-order wetting transitions, while for $g<-2$, the solid curve represents the locus of continuous wetting transitions. The point $g=-2, h_{1}=2$ is a (surface) tricritical point.

The surface tensions $\sigma^{\alpha \gamma}$ and $\sigma^{\alpha \gamma^{*}}$ of the two coexisting surface phases $\alpha \gamma$ and $\alpha \gamma^{*}$, respectively, are obtained by solving the following Euler-Lagrange equation, associated with the minimization of Eq. (2.6) with respect to $\rho$ :

$$
\frac{\partial^{2} \rho}{\partial z^{2}}=f^{\prime}(\rho)
$$

and the appropriate boundary conditions, in the $\alpha$ phase, given by the value of $\rho^{\alpha}$ that minimizes Eq. (2.8), and at the solid substrate

$$
\left.\frac{\partial \rho}{\partial z}\right|_{z=0}=\Phi^{\prime}\left(\rho_{1}\right)
$$

The details of solving Eq. (2.22) have been given by Blokhuis $^{3}$ and we shall here only refer to the results. The analytic expressions for the equilibrium interfacial density profiles are quite elaborate and are given in Eqs. (2.6)-(2.8) and (2.10) of Ref. 3. Inserting these expressions in Eq. (2.6), Blokhuis obtained the analytic expressions for the surface tensions, given in Eqs. (2.9) and (2.10) of Ref. 3.

By comparing the corresponding surface tensions of the $\alpha \gamma$ and $\alpha \gamma^{*}$ interfaces, Blokhuis ${ }^{3}$ determined the prewetting transition, when $\sigma^{\alpha \gamma}=\sigma^{\alpha \gamma^{*}}$, for $g=0$, as a function of $h$ and $h_{1}$. Figure 3 in Ref. 3 shows the prewetting line in the $h-h_{1}$ thermodynamic space. Furthermore, he determined an approximate analytic prewetting line $h_{1}(h)$ close to the wetting transition,

$$
h_{1, \text { prewet }}=h_{1}^{w}+\frac{h}{\Delta \rho_{1}^{w}}\left[\log \left(\frac{4\left(h_{1}^{w}\right)^{2}}{h}\right)+1\right]+O\left(h^{2}\right),
$$

where $h_{1}^{w}=(2 \sqrt{3}-3)^{1 / 2} \approx 0.68125$ is the value of $h_{1}$ at the wetting transition (for $g=0$ ) and $\Delta \rho_{1}^{w} \approx 1.8612$ is the corresponding density difference between the $\alpha \gamma^{*}$ and $\alpha \gamma$ surface phases, at the substrate.

In the next section, we present the calculations of the line tension of the three-phase contact line and of the boundary tension of the boundary between two surface phases, as the first-order wetting transition is approached from two different directions - along the partially wet states of the threephase coexistence curve (the solid curve below the $W$ point in Fig. 3) and along the prewetting line (dashed curve in Fig. 3 ). The calculations are done for $g=0$, by varying $h_{1}$ (and $h$, for the prewetting case). The (first-order) wetting transition, for $g=0$, occurs at $h_{1}^{w}=(2 \sqrt{3}-3)^{1 / 2}($ and $\left.h=0)\right)^{3}$

\section{LINE TENSION}

The analogous expression to Eq. (1.4) for the line tension $\tau$ of the three-phase contact line, using the model free energy from Sec. II, is given by the following form: ${ }^{14}$

$\tau=\min _{\rho} \int_{-\infty}^{\infty} d x \int_{0}^{\infty} d z \Psi(\rho)+\int_{-\infty}^{\infty} d x\left[\Phi\left(\rho_{1}\right)-\Sigma(x)\right]$,

where the functions $\Psi(\rho)$ and $\Phi\left(\rho_{1}\right)$ are as in Eqs. (2.2) and (2.5) respectively, and $\Sigma(x)$ is given by

$$
\Sigma(x)=\sigma^{\alpha \gamma} H(-x)+\left(\sigma^{\beta \gamma}+\frac{\sigma^{\alpha \beta}}{\cos \theta}\right) H(x) .
$$

The unit step function $H(x)$ is defined as follows: 


$$
H(x)= \begin{cases}1, & x \geqslant 0 \\ 0, & x<0\end{cases}
$$

The function $\rho \equiv \rho(x, z)$ is the local density in the fluid phases and $\rho_{1} \equiv \rho(x, z=0)$ is the fluid density at the solid substrate. The surface tensions $\sigma^{\alpha \gamma}, \sigma^{\beta \gamma}$, and $\sigma^{\alpha \beta}$, and the contact angle $\theta$ are defined as functions of the parameters $g$ and $h_{1}$ as in Eqs. (2.18)-(2.20) and (2.21), respectively. The gradient operator $\boldsymbol{\nabla}$ is two dimensional in the $x$ and $z$ directions, which are perpendicular to the three-phase contact line. There is no variation in the density in the direction parallel to the three-phase contact line.

The boundary tension $\tau_{b}$ of the one-dimensional boundary between two coexisting surface phases is given as an extension of Eq. (3.1)

$$
\tau_{b}=\min _{\rho} \int_{-\infty}^{\infty} d x \int_{0}^{\infty} d z \Psi_{b}(\rho)+\int_{-\infty}^{\infty} d x\left[\Phi\left(\rho_{1}\right)-\sigma\right]
$$

where $\Psi_{b}(\rho)$ and $\Phi\left(\rho_{1}\right)$ are as in Eqs. (2.7) and (2.5) respectively, and where $\sigma$ is the surface tension of the two coexisting interfaces $\left(\sigma=\sigma^{\alpha \gamma}=\sigma^{\alpha \gamma^{*}}\right)$ [Eq. (2.6)].

In earlier works, ${ }^{3-5}$ the problem of minimizing functionals of the forms (3.1) and (3.3), with respect to a twodimensional function $\rho(x, z)$, was approached by approximating $\rho(x, z)$ with a qualitatively correct functional form with variational parameters, whose values are obtained by minimization. The obtained values for $\tau$ and $\tau_{b}$ are then upper bounds to the line and boundary tensions, respectively.

Proceeding with this approach, we assume the following functional form for the density $\rho(x, z)$, for the partially wet system $(h=0)$,

$$
\begin{aligned}
\rho(x, z)= & -\tanh \left\{z \cos [\theta f(x)]-x \sin [\theta f(x)]-z_{0}[1-f(x)]\right\} \\
& +\left[\operatorname{coth}\left(z+z_{1}\right)-1\right] f(x),
\end{aligned}
$$

with

$$
f(x)=\frac{1}{2} \tanh \left(\frac{x-x_{0}}{w}\right)+\frac{1}{2},
$$

where $x_{0}$ and $w$ are the two variational parameters, denoting the location and width of the contact region, respectively.

The density profile $\rho(x, z)$ in Eq. (3.4) becomes the exact profile of Eq. (2.12) as $x \rightarrow-\infty$; the exact profile of Eq. (2.13) as $x \rightarrow+\infty$, for fixed $z$ and the exact profile of Eq. (2.14) as $x, z \rightarrow+\infty$, for fixed $t$. Consequently, the exact expressions for the surface tensions $\sigma^{\alpha \gamma}, \sigma^{\beta \gamma}$, and $\sigma^{\alpha \beta}$ in Eqs. (2.18)-(2.20) are retrieved. The densities of the bulk $\alpha$ and $\beta$ phases, $\rho^{\alpha}=-1$ and $\rho^{\beta}=1$ emerge from Eq. (3.4) as well. However, expression (3.4) is approximate everywhere else.

With $\rho(x, z)$ as in Eq. (3.4), the minimization of Eq. (3.1) with respect to $x_{0}$ and $w$, for given values of the model's parameters $g$ and $h_{1}$, yields an upper bound to the line tension. These results are shown in Fig. 6 as solid squares.

Using the same approach, Blokhuis ${ }^{3}$ calculated the upper bound to the boundary tension. These results are plotted as open squares in Fig. 6. Blokhuis determined that the value of the boundary tension at the wetting transition is 1.602 and that close to the wetting transition, $\tau_{b}=1.602-4.503 h^{1 / 2}$.

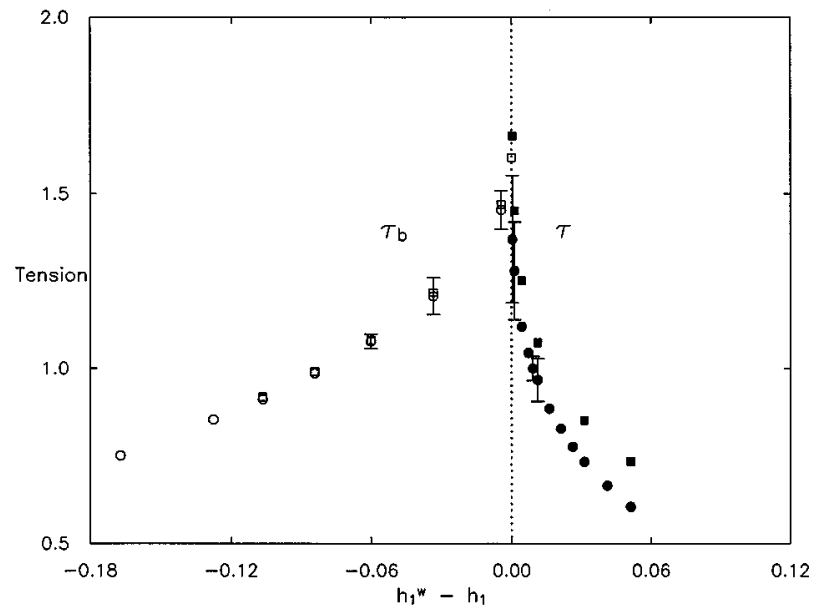

FIG. 6. A plot of the approximate (squares) and exact (circles) line and boundary tensions vs $h_{1}^{w}-h_{1}$ for $g=0$ and $h_{1}^{w}=0.68125$. The wetting transition occurs at $h_{1}^{w}-h_{1}=0$. The solid circles and squares represent the data for the line tension, while the open ones represent the data for the boundary tension. The approximate results are upper bounds to the exact line and boundary tensions. The error bars are the standard deviations from the mean.

In Fig. 6, the data are plotted against $h_{1}^{w}-h_{1}$. In the same figure, we show the numerically exact values for $\tau$ and $\tau_{b}$ (solid and open circles, respectively), which will be determined below. The approximate calculations do indeed yield upper bounds to the line and boundary tensions. The difference between the exact boundary tension and the upper bound to the boundary tension is at most $1 \%$ and usually even smaller, suggesting that the minimizing density profile is close to the functional form of the approximate density profile. ${ }^{3}$ It should be kept in mind though, that small deviations in the density profile away from the minimizing density profile only contribute quadratically to a change in the value of the boundary tension. Next, we proceed to calculate $\tau$ and $\tau_{b}$ exactly using a multigrid algorithm.

The line tension $\tau$ is obtained by minimizing Eq. (3.1) to yield the Euler-Lagrange equation

$$
\nabla_{\rho}^{2}-2 \rho^{3}+2 \rho=0,
$$

where $\boldsymbol{\nabla}$ is two dimensional, and the following boundary condition:

$$
-h_{1}-\left.g \rho\right|_{z=0}=\left.\frac{\partial \rho}{\partial z}\right|_{z=0} .
$$

Three more boundary conditions are given by

$$
\rho= \begin{cases}-1, & z \rightarrow+\infty \\ -\tanh \left(z-z_{0}\right), & x \rightarrow-\infty, \\ -\tanh (t)-1+\operatorname{coth}\left(z+z_{1}\right), & x \rightarrow+\infty\end{cases}
$$

where $z_{0}$ and $z_{1}$ are defined in Eqs. (2.16) and (2.17), respectively, and $t$ is defined in Eq. (2.15).

Since the integral in Eq. (3.1) converges sufficiently fast as $x \rightarrow \pm \infty$ and $z \rightarrow+\infty$, we can substitute it with an integral over a large, but finite domain. The sides of this irregular domain must be perpendicular to the three two-phase inter- 


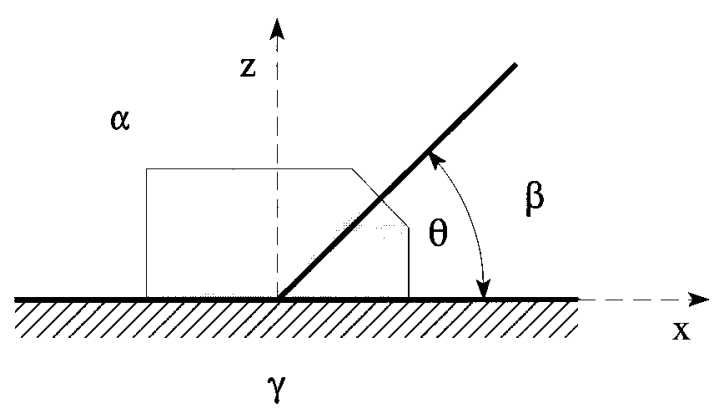

FIG. 7. A side view of two fluid phases $\alpha$ and $\beta$ on a substrate $\gamma$ in a partially wet state. $\theta$ is the contact angle that the $\beta$ phase forms with the substrate $\gamma$. The $x$ axis is parallel to the substrate, while the $z$ axis is perpendicular to it. The boxed region is the area over which the integration in Eq. (3.1) is performed to calculate the line tension.

faces in order for the line tension $\tau$ to be invariant to the change of location of the three-phase contact line (Fig. 7). It is in this domain that the Euler-Lagrange equation (3.6) needs to be solved for the density $\rho$. However, in practice, the Euler-Lagrange equation is solved for the density in a rectangular or square domain, with the line tension $\tau$ calculated from Eq. (3.1) by substituting the value of the density in the irregular domain only.

The Euler-Lagrange equation (3.6) is a nonlinear partial differential equation with one Neumann condition (3.7) and three Dirichlet boundary conditions (3.8). Traditional methods of solution use relaxation methods (such as the Jacobi or Gauss-Seidel methods) to iteratively solve the discretized form of Eq. (3.6) for the density $\rho$. However, the relaxation becomes very slowly convergent in the limit when the grid spacing $h_{s} \rightarrow 0$. In order to speed up the convergence of a traditional relaxation method, a multigrid algorithm is used. The idea of this method is to transfer the solution $\rho$ from a fine grid onto a coarser grid via a restriction operator, once the convergence rate of the iterative (smoothing) method on the fine grid becomes too slow, while it is still good on the coarser grid. This process is repeated until the coarsest grid is reached, where usually the solution can be obtained exactly. At that point, the solution $\rho$ is brought back onto the finest grid via a prolongation operator.

We use a nonlinear multigrid method-the full approximation storage algorithm (FAS) ${ }^{18}$ The smoothing at each grid level is achieved with a red-black Gauss-Seidel relaxation method. The prolongation operator is a bilinear interpolation and the restriction operator uses a half-weighting restriction. We determine the two-dimensional density profile $\rho(x, z)$ in two domain geometries: a square domain of 513 $\times 513$ grid points and a rectangular domain of $513 \times 257$ grid points on the finest grid, keeping the grid spacing $h_{s}$ constant. The advantage of the rectangular domain over the square one is that the number of iterations necessary for convergence is much smaller. The number of grid points in one dimension, on the finest grid, is $2^{N G}+1$, where $N G$ is the number of discretization grids. Because of the restriction on the number of points on the finest grid, given $N G$ and the domain size, the spacing $h_{s}$ is not independent of the domain

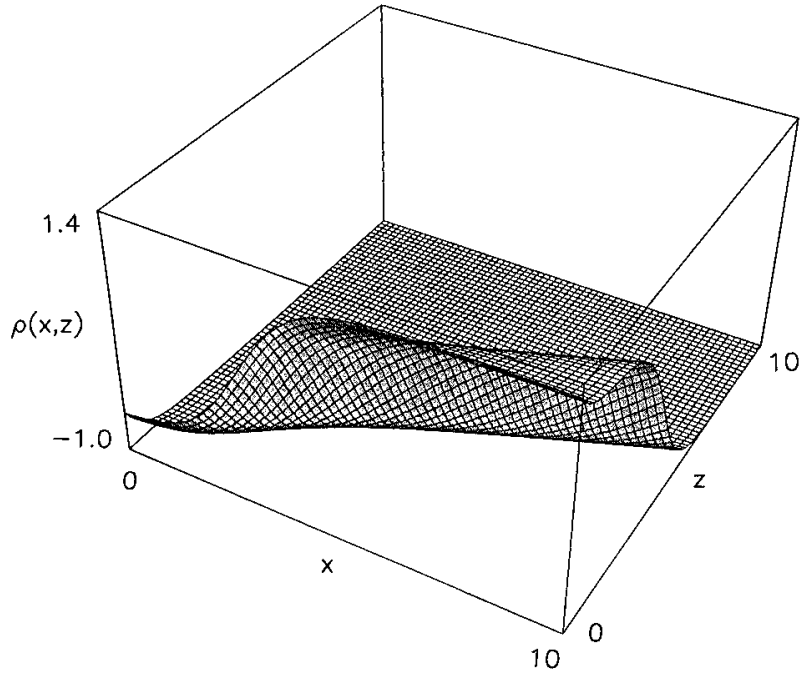

FIG. 8. Density profile $\rho(x, z)$ of the partially wet system at $g=0$ and $h_{1}=0.63$.

size. The largest spacing $h_{s}$ used was $h_{s}=0.045$. In Fig. 8, we show an example of a two-dimensional density profile $\rho(x, z)$ in a partially wet system, obtained as the solution of the Euler-Lagrange equation (3.6), using the multigrid algorithm, for $g=0$ and $h_{1}=0.63$. From such a density profile, the line tension $\tau$ is obtained from Eq. (3.1). As we shall demonstrate below, highly accurate results for the line tension far from the wetting transition are obtained, while the accuracy decreases as the wetting transition is approached.

The boundary tension $\tau_{b}$ is calculated by minimizing Eq. (3.3) with respect to $\rho$ to obtain the Euler-Lagrange equation

$$
\nabla_{\rho}^{2}-2 \rho^{3}+2 \rho-h=0
$$

where $\boldsymbol{\nabla}$ is two dimensional, and the following boundary condition on the solid substrate:

$$
-h_{1}-\left.g \rho\right|_{z=0}=\left.\frac{\partial \rho}{\partial z}\right|_{z=0} .
$$

Three more boundary conditions are given by Eq. (2.3) in Ref. 3 for the density in the $\alpha$ phase and Eq. (2.6) [along with Eqs. (2.7)-(2.10)] in Ref. 3 for the density profiles of the $\alpha \gamma$ and $\alpha \gamma^{*}$ interfaces. We do not want to reproduce them here due to their length and awkwardness, so we refer the reader to Ref. 3 for the explicit expressions.

The integrand in Eq. (3.3) approaches 0 sufficiently fast as $x \rightarrow \pm \infty$ and $z \rightarrow+\infty$ for the integral to converge. Therefore, we can substitute the limits of the integration with those of a large, but finite square or rectangular domain containing the one-dimensional boundary and whose two sides are perpendicular to the two surface phases (Fig. 9). The boundary tension is then determined by solving the Euler-Lagrange equation (3.9) in such a domain. The FAS multigrid algorithm is used in this case as well. The largest square domain that we use consists of $257 \times 257$ grid points, while the largest rectangular domain has $513 \times 257$ points. The largest spacing $h_{s}$ is 0.078 . In Fig. 10, we show an example of a two-dimensional density profile $\rho(x, z)$ in a system of two 


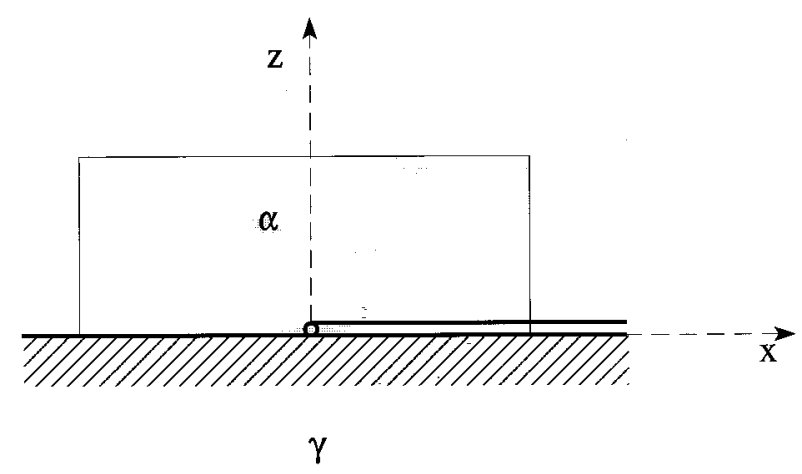

FIG. 9. A side view of two coexisting surface phases at the prewetting transition. The $x$ axis is parallel to the substrate, while the $z$ axis is perpendicular to it. The rectangular region represents the area over which the integration in Eq. (3.3) is done to calculate the boundary tension.

surface phases at coexistence for $g=0, h_{1}=1.202$, and $h=0.3$. Substituting that density in Eq. (3.3), one obtains the boundary tension $\tau_{b}$. Highly accurate results are obtained for the boundary tension away from the wetting transition, while the accuracy decreases close to the wetting transition.

In Fig. 11, two sets of data are plotted against $h_{1}^{w}-h_{1}$, where $h_{1}^{w}$ is the value of the parameter $h_{1}$ at the wetting transition [for the plotted data, $g=0$ and $h_{1}^{w} \approx 0.68125$ (Ref. 3)]. The data on the right curve represent the values of the exact line tension of the three-phase contact line calculated with the multigrid algorithm. The smallest angle $\theta$ for which we obtain a result is $1.51^{\circ}$, which is much smaller than the $13^{\circ}$ angle obtained by Szleifer and Widom ${ }^{5}$ in their calculation of $\tau$. The data points on the left curve of Fig. 11 represent the values of the exact boundary tension $\tau_{b}$. As the wetting transition is approached $\left(h_{1}^{w}-h_{1} \rightarrow 0\right)$, the line tension $\tau$ and the boundary tension $\tau_{b}$ increase in magnitude. To obtain the value of $\tau$ and $\tau_{b}$ at the wetting transition (the two

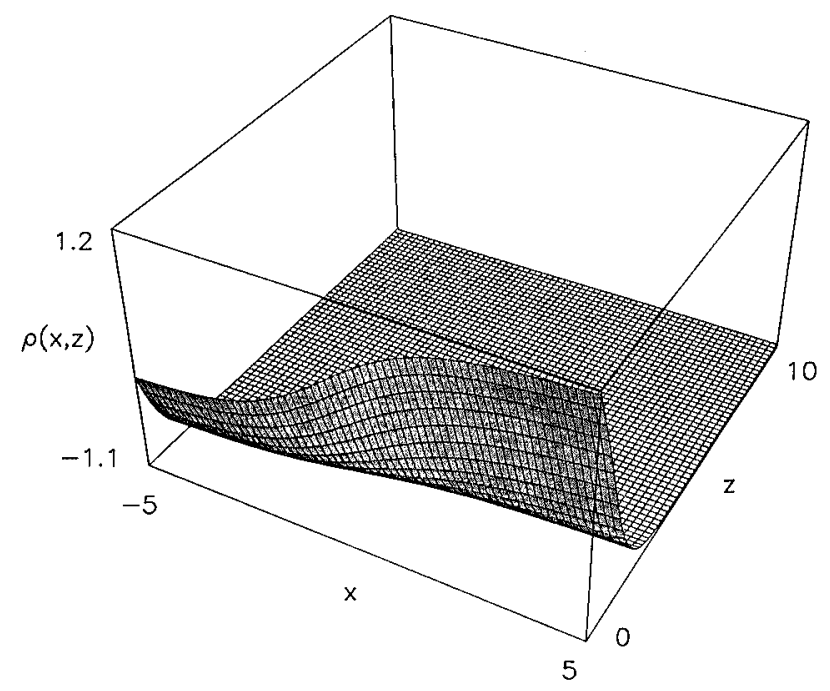

FIG. 10. Density profile $\rho(x, z)$ of the system at the prewetting transition at $g=0, h_{1}=1.202$, and $h=0.3$.

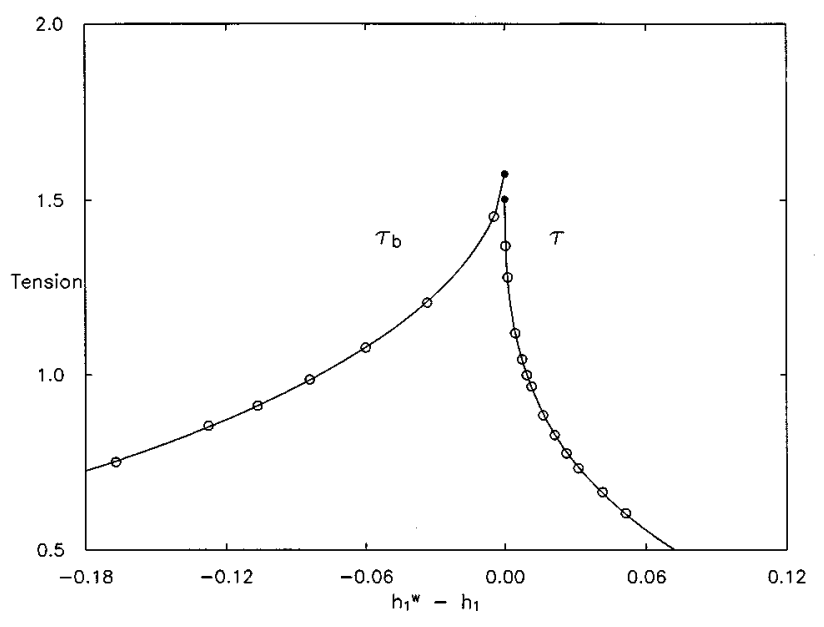

FIG. 11. A plot of the (numerically) exact line and boundary tensions vs $h_{1}^{w}-h_{1}$ for $g=0$ and $h_{1}^{w}=0.68125$. The wetting transition occurs at $h_{1}^{w}-h_{1}=0$. The data are fitted with functional forms described in the text. The solid circles at the top of the curves denote the values of the line and boundary tensions at the wetting transition obtained from the fits. At the wetting transition, the tensions are finite and equal (within numerical errors): $\tau_{w}=1.50$ and $\tau_{b, w}=1.57$.

solid circles on the curves in Fig. 11), we fit the calculated values for the line and boundary tensions with the following expressions:

$$
\tau=\tau_{w}-\tau_{-}\left(h_{1}^{w}-h_{1}\right)^{1 / 2} \log \left(\frac{1}{\left(h_{1}^{w}-h_{1}\right)}\right)+c_{1}\left(h_{1}^{w}-h_{1}\right)^{1 / 2}
$$

and

$$
\tau_{b}=\tau_{b, w}-\tau_{+, h} h^{1 / 2}+c_{2} h,
$$

where $\tau_{w}, \tau_{-}$, and $c_{1}$ are fitting parameters for the line tension, and $\tau_{b, w}, \tau_{+, h}$, and $c_{2}$ are fitting parameters for the boundary tension. The best fits are obtained with the following values of the fitting parameters:

$$
\begin{aligned}
& \tau_{w}=1.50, \quad \tau_{-}=0.725, \quad c_{1}=-1.83, \\
& \tau_{b, w}=1.57, \quad \tau_{+, h}=3.97, \quad c_{2}=3.28 .
\end{aligned}
$$

The forms in Eqs. (3.11) and (3.12) have been derived analytically by Indekeu ${ }^{6}$ using an interface displacement model, and by Blokhuis, ${ }^{3,15}$ within the van der Waals theory. In their work, the second correction terms in Eqs. (3.11) and (3.12) are omitted. Here, however, since very close to the wetting transition data are extremely difficult to obtain, we need to rely on data further away from the wetting transition to obtain a reasonable fit, and so we include the second correction terms. The fact that Indekeu and Blokhuis obtained the same functional forms for $\tau$ and $\tau_{b}$ near the wetting transition with quite different approaches suggests that Eqs. (3.11) and (3.12) are the appropriate fitting forms. In order to compare the values of $\tau$ and $\tau_{b}$, the values of $\tau_{b}$ are plotted, in Fig. 11, as a function of $h_{1}^{w}-h_{1}$ after making use of Eq. (3.12) and the equation for the prewetting line $h_{1}(h) .^{3}$

The line and boundary tensions approach a finite value at the wetting transition with an infinite slope. At the wetting transition, the line tension $\tau=1.50$, is almost equal (within 
the numerical error) to the value of the boundary tension $\tau_{b}=1.57$, as is expected. ${ }^{16}$ The small difference between the values for $\tau$ and $\tau_{b}$ at the wetting transition attests to the accuracy of the multigrid method.

We now make further comparisons of our results with those by Indekeu ${ }^{6}$ and Blokhuis. ${ }^{3}$ Inverting and substituting the approximate analytic prewetting line $h_{1}(h)$ [Eq. (2.24)] in Eq. (3.12), as $h \rightarrow 0$ so that only the first order correction in Eq. (3.12) is used, the following expression for $\tau_{b}$ as a function of $h_{1}^{w}-h_{1}$ is obtained:

$$
\tau_{b}=\tau_{b, w}-\tau_{+}\left(h_{1}-h_{1}^{w}\right)^{1 / 2} \log ^{-1 / 2}\left[\frac{1}{\left(h_{1}-h_{1}^{w}\right)}\right] .
$$

From Eqs. (3.12), (2.24), and (3.13), for small $h$, so that only the first two terms of Eq. (3.12) are used, we obtain

$$
\tau_{+, h}\left(\Delta \rho_{1}^{w}\right)^{1 / 2}=\tau_{+} .
$$

The ratio $Q=\tau_{+} / \tau_{-}$is a universal amplitude ratio. ${ }^{13}$ Within the interface displacement model ${ }^{10}$ and the van der Waals theory, ${ }^{3,15} Q=5.515$. From the fits of the line and boundary tensions, $\tau_{-}=0.725$ and $\tau_{+, h}=3.97$. Using these values and Eq. (3.14), we obtain for the universal amplitude ratio $Q=7.47$. We will discuss the apparent disagreement between these two values in the Discussion section.

As a test of the accuracy of the line and boundary tensions obtained with the multigrid algorithm, we calculate $\tau$ and $\tau_{b}$ using expressions analogous to the Kerins-Boiteux formula, ${ }^{19}$ applied to a two-fluid system on a solid substrate. The line tension $\tau^{\mathrm{K}-\mathrm{B}}$ is (Appendix B)

$$
\tau^{\mathrm{K}-\mathrm{B}}=\int_{-\infty}^{\infty} d x \int_{0}^{\infty} d z\left[\frac{1}{2}(\boldsymbol{\nabla} \rho)^{2}-f(\rho)\right],
$$

and the boundary tension $\tau_{b}^{\mathrm{K}-\mathrm{B}}$ is (Appendix A)

$$
\tau_{b}^{\mathrm{K}-\mathrm{B}}=\int_{-\infty}^{\infty} d x \int_{0}^{\infty} d z\left[\frac{1}{2}(\boldsymbol{\nabla} \rho)^{2}-f(\rho)+f\left(\rho^{\alpha}\right)\right],
$$

where $f(\rho)$ is defined as in Eq. (2.3) for the partially wet case (3.15) and as in Eq. (2.8) for the prewet case (3.16). Another formula for the boundary tension $\tau_{b}$ is also obtained (Appendix A)

$$
\tau_{b}=\int_{-\infty}^{\infty} d x \int_{0}^{\infty} d z\left(\frac{\partial \rho}{\partial x}\right)^{2} .
$$

The integrals in Eqs. (3.15)-(3.17) are not variational integrals. The density $\rho$ in these expressions is the equilibrium profile. The line tension $\tau^{\mathrm{K}-\mathrm{B}}$ obtained from Eq. (3.15) and the boundary tensions $\tau_{b}^{\mathrm{K}-\mathrm{B}}$ obtained from Eq. (3.16) and $\tau_{b}$ from Eq. (3.17) are not extrema when $\rho$ is the equilibrium profile.

The difference in the values for the line tensions obtained from Eqs. (3.1) and (3.15) describes, qualitatively, how close the density profile $\rho$, obtained with the multigrid algorithm, is to its equilibrium profile. When the difference is small, the density profile is close to its equilibrium profile and the line tension is close to its exact value. We have used the line tension values from Eqs. (3.1) and (3.15) to determine the average line tension and its standard deviation.
These error bars are centered at the values of the line tension from the multigrid method, as shown in Fig. 6. We have chosen to keep the line tension values as those determined by the multigrid method, rather than the average line tension from Eqs. (3.1) and (3.15), since the density profile $\rho$, obtained from the multigrid algorithm, is, within the numerical accuracy, the solution of the Euler-Lagrange equation (3.6) and hence gives the best estimate of the line tension. When no error bars are shown in Fig. 6, the values of $\tau$ from Eqs. (3.1) and (3.15) are essentially identical. In Fig. 6, far from the wetting transition, there is essentially no difference between the line tensions obtained with the multigrid algorithm (3.1) and the ones obtained from the Kerins-Boiteux formula (3.15): the density profile $\rho$ obtained as the solution to Eq. (3.6) is the equilibrium profile. As the wetting transition is approached, discrepancies start to occur, and very close to the wetting transition, they are significant, as demonstrated by the large error bars. Such discrepancies occur because of the increase in the inhomogeneous area associated with the three-phase contact line as the wetting transition is approached, and the subsequent difficulty in obtaining accurate results for large domains, i.e., large spacing $h_{s}$. This problem can be solved by using fast computers with large memories to accommodate larger grid sizes, necessary to obtain accurate results.

In the same figure (Fig. 6), we show the standard deviation from the average boundary tension calculated from Eqs. (3.3), (3.16), and (3.17) as error bars centered around the values of $\tau_{b}$ from the multigrid algorithm. Here again, the same kind of analysis and conclusions apply as for the partially wet case.

\section{SURFACE POTENTIAL}

An important quantity for the understanding of the wetting behavior of a liquid phase on a substrate is the so-called surface potential. ${ }^{20-23}$ The surface potential is usually given as a function of the height of the liquid-vapor interface (the $\alpha \beta$ interface) above the substrate, and measures the surface free energy when the interface is constrained to be at a certain height $l$ different from its equilibrium height $l_{\text {eq }}$. The height $l$ is defined as the value of $z$ where the density profile crosses the value zero. The system is as the system depicted in Fig. 2, but now one has to keep in mind that the location of the $\alpha \beta$ interface may vary. Different forms of the surface potential can be derived using different methods of constraining the interface at a certain height. In this section, we will briefly review some of these forms for the surface potential and compare them with the surface potential that is derived from our numerical results for the density profile along the prewetting line. Furthermore, we present the numerical form of the surface tension of the liquid-vapor interface as a function of the height $l, \sigma_{0}(l)$. The precise definition of $\sigma_{0}(l)$ is given below.

The surface potential is derived from the surface free energy [cf. Eqs. (2.6) and (2.7)]

$$
F_{s}[\rho]=\int_{0}^{\infty} d z\left[\frac{1}{2}\left(\frac{\partial \rho}{\partial z}\right)^{2}+f(\rho)-f\left(\rho^{\alpha}\right)\right]+\Phi\left(\rho_{1}\right) .
$$


Two forms of the surface potential were recently proposed. The first one is by Fisher and $\mathrm{Jin}^{22}$ and is derived by minimizing Eq. (4.1) and adding a Lagrange multiplier of the form $\lambda \rho(z) \delta(z-l)$ to obtain

$$
V_{\mathrm{FJ}}(l) \equiv \min _{\rho}\left[F_{s}(\rho)+\lambda \rho(z) \delta(z-l)-\sigma\right] .
$$

The addition of a Lagrange multiplier of this form fixes the density to be zero at height $l$. In the above expression, we have also subtracted the surface free energy $\sigma$ defined as the equilibrium surface free energy. The surface potential then has the property that $V\left(l_{\text {eq }}\right)=0$. The density profile that results from the minimization with the above constraint is, however, nonanalytic. The first derivative with respect to $z$ is discontinuous at $z=l$ whenever $l$ is not equal to $l_{\text {eq }}$.

A second form for the surface potential was proposed by Bukman et al. ${ }^{23}$ In their analysis, they choose to constrain the surface free energy in Eq. (4.1) by fixing the adsorption. Hence,

$$
V_{B}(l) \equiv \min _{\rho}\left[F_{s}(\rho)+\lambda \int_{0}^{\infty} d z\left(\rho-\rho^{\alpha}\right)-\sigma\right] .
$$

Unfortunately, the resulting density profile does not become equal to the vapor density $\rho^{\alpha}$ when $z$ goes to infinity. The authors resolve this by allowing a discontinuity in the second derivative in at most one point. As in the previous case, the profile is thus nonanalytic. An important advantage of this approach is that it is very natural to constrain the height of the interface by fixing the adsorption. Also, the adsorption itself is physically a much more relevant variable than the height $l$ which is sometimes, as we will see later, ill defined.

A third form of the surface potential is defined by the following constraint: ${ }^{22}$

$$
V_{2}(l) \equiv \min _{\rho}\left[F_{s}(\rho)+\lambda \int_{0}^{\infty} d z\left(\rho-\rho^{\alpha}\right)^{2}-\sigma\right] .
$$

Although the addition of the term with the Lagrange multiplier of this form is not well motivated physically, the surface potential thus defined does not suffer the aforementioned drawbacks of the other surface potentials. The density profile is analytic and, furthermore, the surface potential can be calculated explicitly. The above surface potential was used by Blokhuis ${ }^{3}$ to calculate the upper bound to the boundary tension at the wetting transition and along the prewetting line.

The surface potential is an important quantity whenever one is interested in the behavior of the wetting system when the location of the liquid-vapor interface is somehow forced out of its equilibrium location. For instance, the surface potential is important to describe thermal fluctuations of the liquid-vapor interface or, also, for the calculation of the line tension or boundary tension. In the latter case, the surface potential has two equal minima when the height equals the height of the thin or thick films, indicative of the coexistence of the thin and thick films along the prewetting line. In fact, an essential part of the boundary tension is due to the fact that the surface potential is larger than zero when $l_{\text {thin }}<l<l_{\text {thick }}$. We now want to turn the analysis around. We have obtained the numerically exact density profiles along the prewetting line and we now want to calculate the form of the surface potential that would have yielded such a profile. It should be kept in mind that this numerically obtained surface potential can only be obtained exactly along the prewetting line while the surface potentials described by the above formalisms can be obtained anywhere in the phase diagram.

In order to obtain, as we will call it $V_{\text {num }}(l)$, we first show how it is derived from the full expression for the free energy per unit length of the boundary line [cf. Eq. (3.3)]

$$
\begin{aligned}
F_{L}[\rho]= & \int_{-\infty}^{\infty} d x \int_{0}^{\infty} d z\left[\frac{1}{2}\left(\frac{\partial \rho}{\partial x}\right)^{2}+\frac{1}{2}\left(\frac{\partial \rho}{\partial z}\right)^{2}+f(\rho)\right. \\
& \left.-f\left(\rho^{\alpha}\right)\right]+\int_{-\infty}^{\infty} d x\left[\Phi\left(\rho_{1}\right)-\sigma\right] .
\end{aligned}
$$

Introducing $l(x)$ as a variable to replace $x$, this is rewritten as $^{3}$

$$
F_{L}[l]=\int_{-\infty}^{\infty} d x\left\{\frac{1}{2} \sigma_{0}(l)\left[\frac{d}{d x} l(x)\right]^{2}+V(l)\right\},
$$

where

$\sigma_{0}(l) \equiv \int_{0}^{\infty} d z\left[\frac{\partial}{\partial l} \rho(l, z)\right]^{2}$,
$\left.V(l) \equiv \int_{0}^{\infty} d z\left\{\frac{1}{2}\left[\frac{\partial}{\partial z} \rho(l, z)\right]^{2}+f(\rho)-f\left(\rho^{\alpha}\right)\right\}+\Phi\left(\rho_{1}\right)-\sigma .7\right)$

The function $\sigma_{0}(l)$ denotes the surface tension against surface area fluctuations of the liquid-vapor interface located at height $l .^{3,22,24}$ When $l$ is large compared to some typical interaction range of the liquid with the substrate $\sigma_{0}(l)$ is expected to become equal to $4 / 3$, the surface tension of the free liquid-vapor interface. The definition of $V(l)$ in Eq. (4.7) is similar to those given in Eqs. (4.2)-(4.4), the only difference being the way in which the liquid-vapor interface is constrained to be at a certain height $l$. Inserting the numerical profile into the above expression for $V(l)$, gives us the desired $V_{\text {num }}(l)$. The result is plotted in Fig. 12, for the case $h=0.2$. As an indication of the numerical accuracy of the obtained $V_{\text {num }}(l)$, we have also calculated $V_{\text {num }}(l)$ using a different but equivalent expression, the derivation of which is given in Appendix A [cf. Eq. (A11)]. The difference between the two results (the open circles and crosses in Fig. 12) is an indication of the numerical accuracy. The line in Fig. 12 is $V_{\mathrm{FJ}}(l)$ and within the numerical accuracy, it is clearly always lower than the numerical results. Notice that we have not plotted $V_{\text {num }}(l)$ for $l<0$ since $l$ is not well-defined in that case. In Fig. 13, we have enlarged the boxed portion of Fig. 12 and included the results of $V_{B}(l)$ (squares) and $V_{2}(l)$ (solid circles) besides $V_{\mathrm{FJ}}(l)$ (triangles). The numerical results are always very close to the three models for $V(l)$ and even quantitatively correct results can be expected when these three models are used to calculate the boundary tension. When the surface potential is plotted as a function of $l$, it is expected, as can also be seen from Figs. 12 and 13, that 


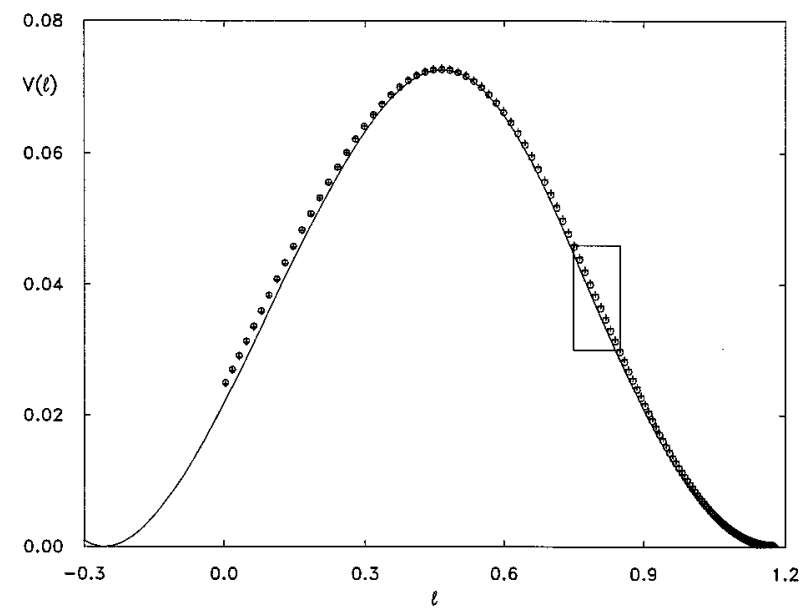

FIG. 12. The surface potential as a function of $l$ for $h=0.2$ on the prewetting line. The solid curve is the surface potential as proposed by Fisher and Jin $V_{\mathrm{FJ}}(l)$. The crosses and open circles are the numerically calculated values of the surface potential. The box shows the region that is depicted in Fig. 13.

$V_{\mathrm{FJ}}(l)$ would give the lowest curve, but when the surface potential is plotted vs the adsorption $\Gamma$, it is expected that the surface potential by Bukman et al. ${ }^{23}$ is going to be the lowest. This is shown in Fig. 14. In this figure, we have plotted the results for the surface potential from Fig. 13 as a function of $\Gamma$ instead of $l$. As expected, the numerical results are close to all three models for the surface potential, but now $V_{B}(\Gamma)$ is the lowest curve.

As a final point, we have numerically calculated $\sigma_{0}(l)$ as given in Eq. (4.7). The result, again for $h=0.2$, is plotted in Fig. 15 (open circles). Also shown (solid curve) is $\sigma_{0}(l)$ that is calculated by inserting the density profile that minimizes Eq. (4.4) into Eq. (4.7). The surface tension of the free liquid-vapor interface is depicted by the broken line. The numerical calculation of $\sigma_{0}(l)$ from the density profile is quite involved; first, the profile has to be numerically differ-

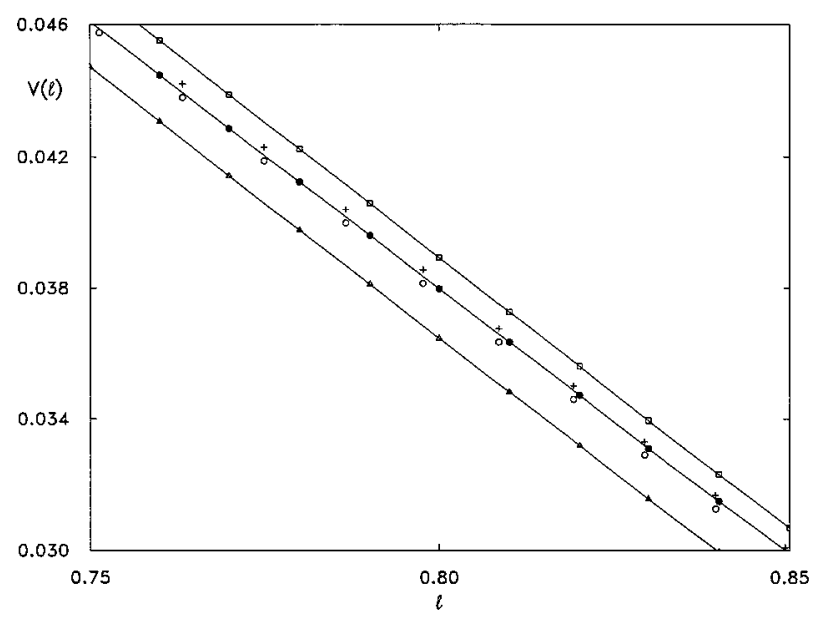

FIG. 13. Details of Fig. 12 (boxed region). The boxes, solid circles, and triangles are the values of the surface potentials $V_{B}(l), V_{2}(l)$, and $V_{\mathrm{FJ}}(l)$, respectively. The crosses and open circles are the numerically calculated values of the surface potential.

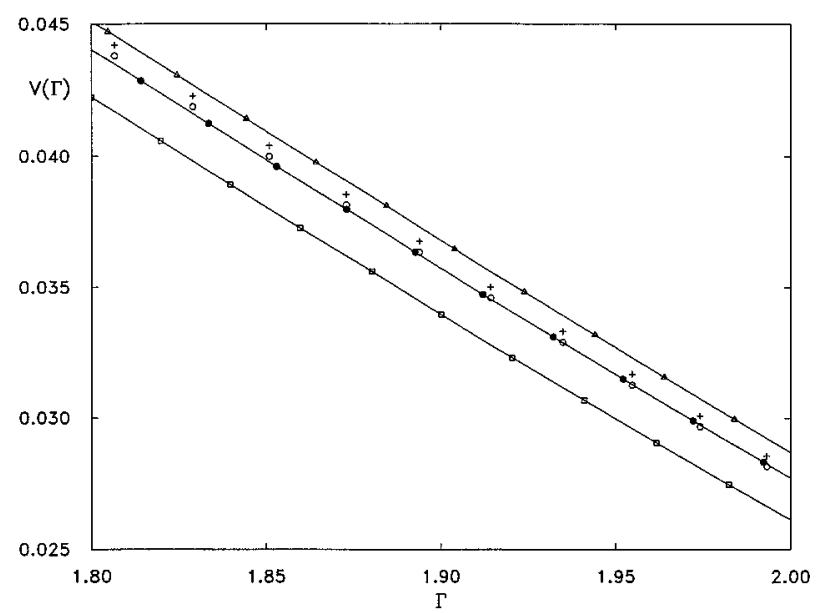

FIG. 14. The surface potential as a function of $\Gamma$ for $h=0.2$ on the prewetting line. The boxes, solid circles, and triangles are the values of the surface potentials $V_{B}(\Gamma), V_{2}(\Gamma)$, and $V_{\mathrm{FJ}}(\Gamma)$, respectively. The crosses and open circles are the numerically calculated values of the surface potential.

entiated with respect to a numerically obtained $l$, then, secondly, the square of the result is numerically integrated over $z$. The numerical error of the result is expected to be of the order of the wiggles for large $l$ in Fig. 15. Only qualitatively does $\sigma_{0}(l)$ derived from Eq. (4.4) agree with $\sigma_{0}(l)$ obtained numerically. For small values of $l$, each surface tension $\sigma_{0}(l)$ of the constrained system is significantly lower than the surface tension of the free liquid-vapor interface, while for larger values, it is significantly higher. As a result it can be seen that the numerically obtained $\sigma_{0}(l)$ at the equilibrium values of $l, l_{\text {thin }}$, and $l_{\text {thick }}$ differ significantly from the surface tension of the free liquid-vapor interface. This implies that if one measures, either in computer simulations or in real experiments, the surface tension against fluctuations of the liquid-vapor interface, that smaller values will be obtained for the thinner films than for the thicker films. It

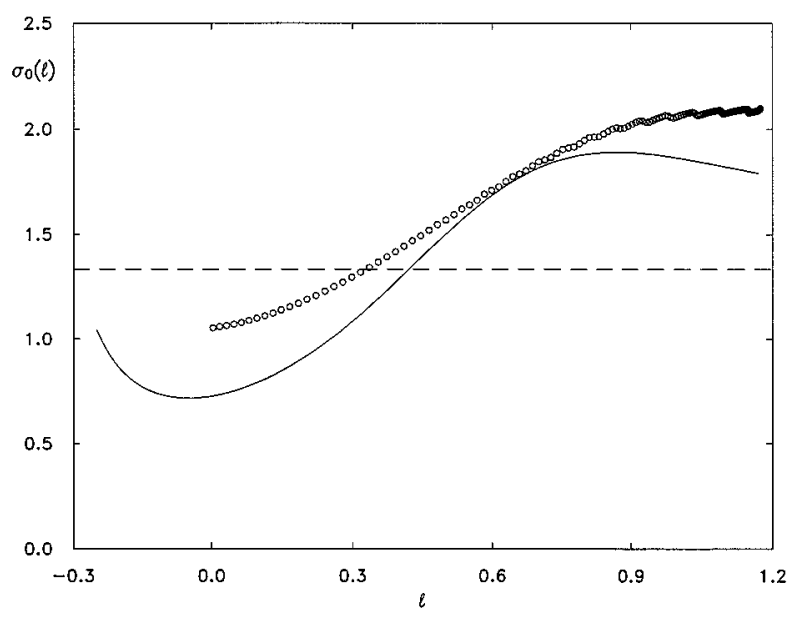

FIG. 15. The surface tension as a function of $l$ for $h=0.2$ on the prewetting line. The solid curve is $\sigma_{0}(l)$ calculated by inserting the density profile that minimizes Eq. (4.4) into Eq. (4.7). The open circles are the numerically calculated values of the surface tension. The horizontal dashed line is at the value of the surface tension of the free liquid-vapor interface. 


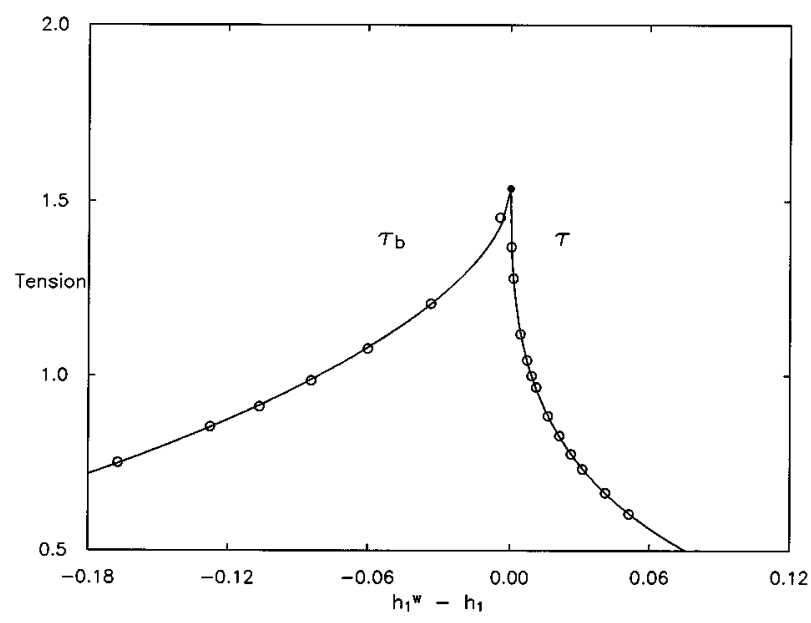

FIG. 16. A plot of the (numerically) exact line and boundary tensions vs $h_{1}^{w}-h_{1}$ for $g=0$ and $h_{1}^{w}=0.68125$. The wetting transition transition occurs at $h_{1}^{w}-h_{1}=0$. The data are fitted by assuming $\tau_{w}=\tau_{b, w}$ and $Q=5.515$ (see the text). At the wetting transition, we find $\tau_{w}=\tau_{b, w}=1.54$

should be remarked that one should not confuse $\sigma_{0}(l)$, which is a property of only the liquid-vapor interface, with the surface tensions of the $\alpha \gamma$ or $\alpha \gamma^{*}$ interface. The latter ones will always be equal to each other along the prewetting line.

\section{DISCUSSION}

In this paper, we have presented (numerically) exact calculations of the line tension of the three-phase contact line and the boundary tension of the boundary between two coexisting surface phases. The approach is a van der Waals-like theory with a model free energy that treats the substrate as a boundary in a manner used by Nakanishi and Fisher. ${ }^{14}$ The line and boundary tensions are equal (within the accuracy of the numerical method), positive, and finite at the wetting transition, and approach it with diverging slopes. These results are obtained by fitting the data for $\tau$ and $\tau_{b}$ from the multigrid calculation with the functional forms determined by Indekeu, ${ }^{6}$ within the interface displacement model, and by Blokhuis, ${ }^{3,15}$ within the van der Waals theory, using approximate expressions for $V(l)$ and $\sigma_{0}$, and $\rho$, respectively. To obtain the same asymptotic forms for $\tau$ and $\tau_{b}$ with two different models, and with approximate methods, suggests that the expressions for the line and boundary tensions do not depend on the details of the system, and supports the universal character of the first order wetting transition. ${ }^{13}$ We check this in this work by comparing the value of the universal amplitude ratio from Refs. 6, 3, and 15, $Q=5.515$, with the one calculated from our data, $Q=7.47$. This is reasonable agreement, considering the difficulty in obtaining accurate values for the line and boundary tensions close to the wetting transition. This conclusion is supported by the following two analyses of different fitting procedures: In Fig. 16, we present a fit of our data for the boundary and line tension calculated from our multigrid algorithm, obtained by assuming that $\tau_{w}=\tau_{b, w}$ and $Q=5.515$. The fit is as accurate as the one in Fig. 11 and we find $\tau_{w}=\tau_{b, w}=1.54$. Furthermore, since the exact values of the boundary tensions are nearly equal to the upper bounds to the boundary tensions (Fig. 6) obtained by Blokhuis ${ }^{3}$ (but still slightly smaller), it might be possible that the exact line and boundary tensions at the wetting transition are close to the value of 1.602, the upper bound to the boundary tension at the wetting transition. Setting $\tau_{w}$ and $\tau_{b, w}$ in Eqs. (3.11) and (3.12) equal to 1.602, and fitting the other four parameters to the numerical data (the line and boundary tensions from the multigrid algorithm), gives $\tau_{-}=1.18$ and $\tau_{+, h}=4.34$, leading to $Q=5.02$. Although the fit is not shown, it is, again, very accurate. Since both of these fitting procedures give excellent results, it is concluded that our numerical data are consistent with $Q=5.515$.

\section{ACKNOWLEDGMENTS}

B. Widom is gratefully acknowledged for discussions of this work and for a careful reading of the manuscript. We would like to thank R. Sribar and I. Szleifer for illuminating discussions and D. J. Bukman for useful comments. The research of E.M.B. has been made possible by a fellowship of the Royal Netherlands Academy of Arts and Sciences. This work was carried out in the research group of B. Widom and is supported by the National Science Foundation and the Cornell University Materials Science Center.

\section{APPENDIX: A KERINS-BOITEUX FORMULA FOR THE BOUNDARY TENSION}

In this appendix, we derive two different, but equivalent, formulas for the boundary tension $\tau_{b}$ along the prewetting line. One of them is a close analog of the Kerins-Boiteux formula ${ }^{19}$ for the line tension derived for systems in which the three phases are all fluid-like. It turns out that the presence of the substrate does not affect the form of the KerinsBoiteux formula in this case.

The boundary tension is given by [cf. Eq. (3.3)]

$$
\begin{aligned}
\tau_{b}= & \int_{-\infty}^{\infty} d x \int_{0}^{\infty} d z\left[\frac{1}{2}\left(\frac{\partial \rho}{\partial x}\right)^{2}+\frac{1}{2}\left(\frac{\partial \rho}{\partial z}\right)^{2}+f(\rho)-f\left(\rho^{\alpha}\right)\right] \\
& +\int_{-\infty}^{\infty} d x\left[\Phi\left(\rho_{1}\right)-\sigma\right]
\end{aligned}
$$

where the density profile $\rho=\rho(x, z)$ is a solution of the Euler-Lagrange equation [cf. Eq. (3.9)]

$$
\frac{\partial^{2} \rho}{\partial x^{2}}+\frac{\partial^{2} \rho}{\partial z^{2}}=f^{\prime}(\rho)
$$

with the boundary condition [cf. Eq. (3.10)]

$$
\left.\frac{\partial \rho}{\partial z}\right|_{z=0}=\Phi^{\prime}\left(\rho_{1}\right)
$$

Multiplying both sides of the Euler-Lagrange equation by $(\partial \rho) /(\partial x)$ and integrating over $z$ from 0 to $\infty$ gives

$$
\int_{0}^{\infty} d z\left[\frac{\partial^{2} \rho}{\partial x^{2}} \frac{\partial \rho}{\partial x}+\frac{\partial^{2} \rho}{\partial z^{2}} \frac{\partial \rho}{\partial x}-f^{\prime}(\rho) \frac{\partial \rho}{\partial x}\right]=0 .
$$


Next, we partially integrate the second term and write the first and third terms as a derivative

$$
\begin{gathered}
\int_{0}^{\infty} d z\left[\frac{1}{2} \frac{\partial}{\partial x}\left(\frac{\partial \rho}{\partial x}\right)^{2}-\frac{\partial \rho}{\partial z} \frac{\partial^{2} \rho}{\partial x \partial z}-\frac{\partial}{\partial x} f(\rho)\right] \\
=\left(-\frac{\partial \rho}{\partial z} \frac{\partial \rho}{\partial x}\right)_{z=0}^{z=\infty}=\Phi^{\prime}\left(\rho_{1}\right) \frac{\partial \rho_{1}}{\partial x},
\end{gathered}
$$

where we have used the boundary condition in Eq. (A3) to derive the last identity.

We now write the second term on the left-hand side as well as the term on the right-hand side of the above equation as a derivative, integrate over $x$ from $x^{\prime}$ to $\infty$, and subsequently drop the prime. We are then finally left with

$$
\int_{0}^{\infty} d z\left[\frac{1}{2}\left(\frac{\partial \rho}{\partial x}\right)^{2}-\frac{1}{2}\left(\frac{\partial \rho}{\partial z}\right)^{2}-f(\rho)+f\left(\rho^{\alpha}\right)\right]=\Phi\left(\rho_{1}\right)-\sigma .
$$

Another important formula can be derived by performing a similar analysis as above, but now multiplying both sides of the Euler-Lagrange equation by $(\partial \rho) /(\partial z)$ and integrating over $x$ from $-\infty$ to $\infty$. We will not do the analysis, which follows exactly the same steps as the analysis above, but we will just give the result

$$
\int_{-\infty}^{\infty} d x\left[-\frac{1}{2}\left(\frac{\partial \rho}{\partial x}\right)^{2}+\frac{1}{2}\left(\frac{\partial \rho}{\partial z}\right)^{2}-f(\rho)+f\left(\rho^{\alpha}\right)\right]=0 .
$$

Integrating Eq. (A6) over $x$ from $-\infty$ to $\infty$ and Eq. (A7) over $z$ from 0 to $\infty$ and adding the results to the expression for the boundary tension in Eq. (A1) leaves us with the analog of the Kerins-Boiteux $(\mathrm{K}-\mathrm{B})$ formula

$$
\tau_{b}^{\mathrm{K}-\mathrm{B}}=\int_{-\infty}^{\infty} d x \int_{0}^{\infty} d z\left[\frac{1}{2}\left(\frac{\partial \rho}{\partial x}\right)^{2}+\frac{1}{2}\left(\frac{\partial \rho}{\partial z}\right)^{2}-f(\rho)+f\left(\rho^{\alpha}\right)\right] .
$$

Another formula that we will use for the boundary tension is obtained by integrating Eq. (A6) over $x$ from $-\infty$ to $\infty$ and adding the result to the expression for the boundary tension in Eq. (A11)

$$
\tau_{b}=\int_{-\infty}^{\infty} d x \int_{0}^{\infty} d z\left(\frac{\partial \rho}{\partial x}\right)^{2}
$$

Although this formula is even simpler than the analog of the Kerins-Boiteux formula in Eq. (A8), it is only valid as an expression for the boundary tension, whereas the formula in Eq. (A8) is also valid for the line tension along partial wetting. This is shown in the next Appendix.

As a final point, we derive an alternative expression for the surface potential. The surface potential is given by the expression in Eq. (4.7)

$$
V(l)=\int_{0}^{\infty} d z\left[\frac{1}{2}\left(\frac{\partial \rho}{\partial z}\right)^{2}+f(\rho)-f\left(\rho^{\alpha}\right)\right]+\Phi\left(\rho_{1}\right)-\sigma .
$$

Inserting Eq. (A6) into this expression gives the much simpler form

$$
V(l)=\int_{0}^{\infty} d z \frac{1}{2}\left(\frac{\partial \rho}{\partial x}\right)^{2}
$$

\section{APPENDIX B: KERINS-BOITEUX FORMULA FOR THE LINE TENSION AT PARTIAL WETTING}

In this appendix, we derive the analog of the KerinsBoiteux formula ${ }^{19}$ for the line tension at partial wetting. In order to derive this formula, it turns out to be more convenient to write the expression for the line tension in polar coordinates $(r, \phi)$ [cf. Eq. (3.1)]

$$
\begin{aligned}
\tau= & \int_{0}^{\pi} d \phi \int_{0}^{\infty} d r r\left[\frac{1}{2}\left(\frac{\partial \rho}{\partial r}\right)^{2}+\frac{1}{2 r^{2}}\left(\frac{\partial \rho}{\partial \phi}\right)^{2}+f(\rho)\right] \\
& +\int_{0}^{\infty} d r\left[\Phi\left(\rho_{1}\right)+\Phi\left(\rho_{\pi}\right)-\sigma^{\alpha \beta}-\sigma^{\alpha \gamma}-\sigma^{\beta \gamma}\right],
\end{aligned}
$$

where $\rho_{1} \equiv \rho(r, \phi=0)$ and $\rho_{\pi} \equiv \rho(r, \phi=\pi)$. The density profile $\rho=\rho(r, \phi)$ is a solution of the Euler-Lagrange equation which, in polar coordinates, reads [cf. Eq. (3.6)]

$$
\frac{\partial^{2} \rho}{\partial r^{2}}+\frac{1}{r} \frac{\partial \rho}{\partial r}+\frac{1}{r^{2}} \frac{\partial^{2} \rho}{\partial \phi^{2}}=f^{\prime}(\rho),
$$

with the boundary conditions [cf. Eq. (3.7)]

$$
\left.\frac{1}{r} \frac{\partial \rho}{\partial \phi}\right|_{\phi=0}=\Phi^{\prime}\left(\rho_{1}\right),\left.\quad \frac{1}{r} \frac{\partial \rho}{\partial \phi}\right|_{\phi=\pi}=-\Phi^{\prime}\left(\rho_{\pi}\right) .
$$

Multiplying both sides of the Euler-Lagrange equation by $(\partial \rho) /(\partial r)$ and integrating over $\phi$ from 0 to $\pi$ gives

$$
\int_{0}^{\pi} d \phi\left[\frac{1}{r} \frac{\partial}{\partial r}\left(r \frac{\partial \rho}{\partial r}\right) \frac{\partial \rho}{\partial r}+\frac{1}{r^{2}} \frac{\partial^{2} \rho}{\partial \phi^{2}} \frac{\partial \rho}{\partial r}-f^{\prime}(\rho) \frac{\partial \rho}{\partial r}\right]=0 .
$$

Next, we partially integrate the second term and write the first and third terms as a derivative

$$
\begin{aligned}
\int_{0}^{\pi} d \phi\left[\frac{1}{2 r^{2}} \frac{\partial}{\partial r}\left(r \frac{\partial \rho}{\partial r}\right)^{2}-\frac{1}{r^{2}} \frac{\partial \rho}{\partial \phi} \frac{\partial^{2} \rho}{\partial r \partial \phi}-\frac{\partial}{\partial r} f(\rho)\right] \\
=\left(-\frac{1}{r^{2}} \frac{\partial \rho}{\partial \phi} \frac{\partial \rho}{\partial r}\right)_{\phi=0}^{\phi=\pi}=\frac{1}{r}\left[\Phi^{\prime}\left(\rho_{1}\right) \frac{\partial \rho_{1}}{\partial r}+\Phi\left(\rho_{\pi}\right) \frac{\partial \rho_{\pi}}{\partial r}\right]
\end{aligned}
$$

where we have used the boundary conditions in Eq. (B3) to derive the last identity.

We now write the second term on the left-hand side as well as the term on the right-hand side of the above equation as a derivative. Next we multiply by $r$, integrate over $r$ from $r^{\prime}$ to $\infty$ and integrate by parts to obtain

$$
\begin{aligned}
\int_{0}^{\pi} & d \phi\left[\frac{r}{2}\left(\frac{\partial \rho}{\partial r}\right)^{2}-\frac{1}{2 r}\left(\frac{\partial \rho}{\partial \phi}\right)^{2}-r f(\rho)\right]_{r=r^{\prime}}^{r=\infty} \\
& +\int_{0}^{\pi} d \phi \int_{r^{\prime}}^{\infty} d r\left[\frac{1}{2}\left(\frac{\partial \rho}{\partial r}\right)^{2}-\frac{1}{2 r^{2}}\left(\frac{\partial \rho}{\partial \phi}\right)^{2}+f(\rho)\right] \\
= & {\left[\Phi\left(\rho_{1}\right)+\Phi\left(\rho_{\pi}\right)\right]_{r=r^{\prime}}^{r=\infty} . }
\end{aligned}
$$


The terms evaluated at $r=\infty$ in the above equation can be combined to give the sum of the three surface tensions. This can be seen more clearly when one introduces $z \equiv R \phi$ and one takes the limit $R \rightarrow \infty$,

$$
\begin{gathered}
\lim _{R \rightarrow \infty} \int_{0}^{\pi R} d z\left[\frac{1}{2}\left(\frac{\partial \rho}{\partial z}\right)^{2}+f(\rho)\right]+\left[\Phi\left(\rho_{1}\right)+\Phi\left(\rho_{\pi}\right)\right]_{r=R} \\
=\sigma^{\alpha \beta}+\sigma^{\alpha \gamma}+\sigma^{\beta \gamma} .
\end{gathered}
$$

With the above equation and after interchanging $r$ and $r^{\prime}$, Eq. (B6) becomes

$$
\begin{aligned}
& \int_{0}^{\pi} d \phi\left[-\frac{r}{2}\left(\frac{\partial \rho}{\partial r}\right)^{2}+\frac{1}{2 r}\left(\frac{\partial \rho}{\partial \phi}\right)^{2}+r f(\rho)\right] \\
& +\int_{0}^{\pi} d \phi \int_{r}^{\infty} d r^{\prime}\left[\frac{1}{2}\left(\frac{\partial \rho}{\partial r^{\prime}}\right)^{2}-\frac{1}{2\left(r^{\prime}\right)^{2}}\left(\frac{\partial \rho}{\partial \phi}\right)^{2}+f(\rho)\right] \\
& =-\Phi\left(\rho_{1}\right)-\Phi\left(\rho_{\pi}\right)+\sigma^{\alpha \beta}+\sigma^{\alpha \gamma}+\sigma^{\beta \gamma}
\end{aligned}
$$

Next we integrate over $r$ from 0 to $\infty$ and use the indentity

$$
\int_{0}^{\infty} d r \int_{r}^{\infty} d r^{\prime} g\left(r^{\prime}\right)=\int_{0}^{\infty} d r r g(r)
$$

to finally write Eq. (B8) as

$$
\begin{aligned}
\int_{0}^{\pi} d \phi \int_{0}^{\infty} d r r[2 f(\rho)]= & \int_{0}^{\infty} d r\left[-\Phi\left(\rho_{1}\right)-\Phi\left(\rho_{\pi}\right)+\sigma^{\alpha \beta}\right. \\
& \left.+\sigma^{\alpha \gamma}+\sigma^{\beta \gamma}\right] .
\end{aligned}
$$

This equation can now be used to rewrite the expression for the line tension in Eq. (B1)

$$
\tau=\int_{0}^{\pi} d \phi \int_{0}^{\infty} d r r\left[\frac{1}{2}\left(\frac{\partial \rho}{\partial r}\right)^{2}+\frac{1}{2 r^{2}}\left(\frac{\partial \rho}{\partial \phi}\right)^{2}-f(\rho)\right] .
$$

This is the analog of the Kerins-Boiteux formula in polar coordinates.

${ }^{1} \mathrm{~J}$. S. Rowlinson and B. Widom, Molecular Theory of Capillarity (Clarendon, Oxford, 1982).

${ }^{2}$ E. H. Hauge and M. Schick, Phys. Rev. B 27, 4288 (1983).

${ }^{3}$ E. M. Blokhuis, Physica A 202, 402 (1994).

${ }^{4}$ S. Perković, I. Szleifer, and B. Widom, Mol. Phys. 80, 729 (1993).

${ }^{5}$ I. Szleifer and B. Widom, Mol. Phys. 75, 925 (1992).

${ }^{6}$ J. O. Indekeu, Physica A 183, 439 (1992).

${ }^{7}$ C. Varea and A. Robledo, Phys. Rev. A 45, 2645 (1992).

${ }^{8}$ C. Varea and A. Robledo, Phys. Rev. E 47, 3772 (1993).

${ }^{9}$ B. Widom and A. S. Clarke, Physica A 168, 149 (1990).

${ }^{10}$ J. O. Indekeu, Int. J. Mod. Phys. B 8, 309 (1994).

${ }^{11}$ H. T. Dobbs and J. O. Indekeu, Physica A 201, 457 (1993).

${ }^{12}$ J. O. Indekeu, G. Backx, and G. Langie, Physica A 196, 335 (1993).

${ }^{13}$ A. Robledo and J. O. Indekeu, Europhys. Lett. 25, 17 (1994).

${ }^{14}$ H. Nakanishi and M. E. Fisher, Phys. Rev. Lett. 49, 1565 (1982).

${ }^{15}$ E. M. Blokhuis (unpublished).

${ }^{16} \mathrm{~B}$. Widom, in Condensed Matter Theories, edited by L. Blum and F. B. Malik (Plenum, New York, 1993), pp. 589-593.

${ }^{17}$ D. J. Durian and C. Franck, Phys. Rev. Lett. 59, 555 (1987), and references therein.

${ }^{18}$ W. H. Press, S. A. Teukolsky, W. T. Vetterling, and B. P. Flannery, Numerical Recipes in Fortran (Cambridge University, Cambridge, 1992).

${ }^{19}$ J. Kerins and M. Boiteux, Physica A 117, 575 (1983).

${ }^{20}$ E. Brézin, B. I. Halperin, and S. Leibler, J. Phys. 44, 775 (1983); Phys. Rev. Lett. 50, 1387 (1983).

${ }^{21}$ R. Lipowsky, D. M. Kroll, and R. K. P. Zia, Phys. Rev. B 27, 4499 (1983).

${ }^{22}$ M. E. Fisher and A. J. Jin, Phys. Rev. B 44, 1430 (1991); Phys. Rev. Lett. 69, 792 (1992); A. J. Jin and M. E. Fisher, Phys. Rev. B 47, 7365 (1993).

${ }^{23}$ D. J. Bukman, J. O. Indekeu, G. Langie, and G. Backx, Phys. Rev. B 47, 1577 (1993).

${ }^{24}$ M. E. Fisher, A. J. Jin, and A. O. Parry, Ber. Bunsenges. Phys. Chem. 98, 357 (1994). 